\title{
Monogenean anchor morphometry: systematic value, phylogenetic signal, and evolution
}

Tsung Fei Khang, Oi Yoon Michelle Soo, Wooi Boon Tan, Lee Hong Susan Lim

Background. Anchors are one of the important attachment appendages for monogenean parasites. Common descent and evolutionary processes have left their mark on anchor morphometry, in the form of patterns of shape and size variation useful for systematic and evolutionary studies. When combined with morphological and molecular data, analysis of anchor morphometry can potentially answer a wide range of biological questions. Materials and Methods. We used data from anchor morphometry, body size and morphology of 13 Ligophorus (Monogenea: Ancyrocephalidae) species infecting two marine mugilid (Teleostei: Mugilidae) fish hosts: Moolgarda buchanani (Bleeker) and Liza subviridis (Valenciennes) from Malaysia. Anchor shape and size data $(n=530)$ were generated using methods of geometric morphometrics. We used 28S rRNA, 18S rRNA, and ITS1 sequence data to infer a maximum likelihood phylogeny. We discriminated species using principal component and cluster analysis of shape data. Adams's $\mathrm{K}_{\text {mult }}$ was used to detect phylogenetic signal in anchor shape. Phylogeny-correlated size and shape changes were investigated using continuous character mapping and directional statistics, respectively. We assessed morphological constraints in anchor morphometry using phylogenetic regression of anchor shape against body size and anchor size. Anchor morphological integration was studied using partial least squares method. The association between copulatory organ morphology and anchor shape and size in phylomorphospace was used to test the Rohde-Hobbs hypothesis. We created monogeneaGM, a new $R$ package that integrates analyses of monogenean anchor geometric morphometric data with morphological and phylogenetic data. Results. We discriminated 12 of the 13 Ligophorus species using anchor shape data. Significant phylogenetic signal was detected in anchor shape. Thus, we discovered new morphological characters based on anchor shaft shape, the length between the inner root point and the outer root point, and the length between the inner root point and the dent point. The species on M. buchanani evolved larger, more robust anchors; those on L. subviridis evolved smaller, more delicate anchors. Anchor shape and size were significantly correlated, suggesting constraints in anchor evolution. Tight integration between the root and the point compartments within anchors confirms the anchor as a single, fully integrated module. The correlation between male 
copulatory organ morphology and size with anchor shape was consistent with predictions from the Rohde-Hobbs hypothesis. Conclusions. Monogenean anchors are tightly integrated structures, and their shape variation correlates strongly with phylogeny, thus underscoring their value for systematic and evolutionary biology studies. Our MonogeneaGM R package provides tools for researchers to mine biological insights from geometric morphometric data of speciose monogenean genera. 
1 Running head: ANCHOR MORPHOMETRY FOR MONOGENEAN SYSTEMATICS

2 Title: Monogenean anchor morphometry: systematic value, phylogenetic signal, and evolution

3 Authors: Tsung Fei Khang ${ }^{1 *}$, Oi Yoon Michelle $\mathrm{Soo}^{2}$, Wooi Boon Tan ${ }^{3}$, Lee Hong Susan Lim ${ }^{2+}$

$4{ }^{1}$ Institute of Mathematical Sciences, Faculty of Science, University of Malaya, 50603 Kuala

5 Lumpur, Malaysia.

$6{ }^{2}$ Institute of Biological Sciences, Faculty of Science, University of Malaya, 50603 Kuala Lumpur,

7 Malaysia.

$8{ }^{3}$ Centre for Tropical Biodiversity Research, University of Malaya, 50603 Kuala Lumpur,

9 Malaysia.

$10 *$ Corresponding author

$11+$ Deceased on 2 August 2014

12 Email: tfkhang@um.edu.my

13 Tel: +60379674171

14 Fax: +60379674143

15 ABSTRACT 
16 Background. Anchors are one of the important attachment appendages for monogenean

17 parasites. Common descent and evolutionary processes have left their mark on anchor

18 morphometry, in the form of patterns of shape and size variation useful for systematic and

19 evolutionary studies. When combined with morphological and molecular data, analysis of anchor

20 morphometry can potentially answer a wide range of biological questions.

21 Materials and Methods. We used data from anchor morphometry, body size and morphology of

2213 Ligophorus (Monogenea: Ancyrocephalidae) species infecting two marine mugilid (Teleostei:

23 Mugilidae) fish hosts: Moolgarda buchanani (Bleeker) and Liza subviridis (Valenciennes) from

24 Malaysia. Anchor shape and size data $(n=530)$ were generated using methods of geometric

25 morphometrics. We used 28S rRNA, 18S rRNA, and ITS1 sequence data to infer a maximum

26 likelihood phylogeny. We discriminated species using principal component and cluster analysis of

27 shape data. Adams's $\mathrm{K}_{\text {mult }}$ was used to detect phylogenetic signal in anchor shape. Phylogeny-

28 correlated size and shape changes were investigated using continuous character mapping and

29 directional statistics, respectively. We assessed morphological constraints in anchor morphometry

30 using phylogenetic regression of anchor shape against body size and anchor size. Anchor

31 morphological integration was studied using partial least squares method. The association

32 between copulatory organ morphology and anchor shape and size in phylomorphospace was used

to test the Rohde-Hobbs hypothesis. We created monogeneaGM, a new R package that integrates

analyses of monogenean anchor geometric morphometric data with morphological and data. Significant phylogenetic signal was detected in anchor shape. Thus, we discovered new morphological characters based on anchor shaft shape, the length between the inner root point and the outer root point, and the length between the inner root point and the dent point. The species on M. buchanani evolved larger, more robust anchors; those on L. subviridis evolved smaller, more delicate anchors. Anchor shape and size were significantly correlated, suggesting 
41 constraints in anchor evolution. Tight integration between the root and the point compartments

42 within anchors confirms the anchor as a single, fully integrated module. The correlation between

43 male copulatory organ morphology and size with anchor shape was consistent with predictions

44 from the Rohde-Hobbs hypothesis. Conclusions. Monogenean anchors are tightly integrated

45 structures, and their shape variation correlates strongly with phylogeny, thus underscoring their

46 value for systematic and evolutionary biology studies. Our MonogeneaGM R package provides

47 tools for researchers to mine biological insights from geometric morphometric data of speciose

48 monogenean genera.

49 Keywords: cluster heat map - geometric morphometrics - Ligophorus - molecular phylogeny -

50 Monogenea - morphological integration - phylogenetic regression - phylomorphospace -

51 principal component analysis - shape and size variation

\section{INTRODUCTION}

53 The Monogenea is a class of flatworms (Platyhelminthes) that are primarily ectoparasites

54 of fish (Whittington, 2005; Hayward, 2005). An adult monogenean parasite has well-developed

55 attachment appendages located at its anterior (prohaptor) and posterior (opisthaptor) regions that

56 help it to resist physical dislodgement from the host. The posterior attachment organs consist of

57 sclerotized hard parts such as hooks, anchors and clamps. Ecologically, monogenean parasites are

58 characterized by their strong host specificity

59 (Whittington et al., 2000). The Monogenea has several desirable features that make it invaluable

60 as a model system for studying evolutionary processes that resulted in its past diversification and 
61 present diversity (Poulin, 2002). Primarily, many of its genera are speciose, morphologically

62 diverse, show well-resolved phylogenies at the familial level (Boeger and Kritsky, 1997, 2001;

63 Mollaret et al., 2000), and samples can be easily obtained in large numbers. It has been used as a

64 model to shed light on ecological forces that shape species community and structure (Rohde,

65 1979; Mouillot et al., 2005; Raeymaekers et al., 2008), to investigate processes leading to

66 speciation and its maintenance (Rohde and Hobbs, 1986; Rohde 1994; de Meeus et al., 1998;

67 Šimková et al., 2002; Hahn et al., 2015; Vanhove and Huyse, 2015), to elucidate host-parasite

68 evolutionary ecology (Desdevises et al., 2002; Huyse et al., 2003; Huyse and Volckaert, 2005;

69 Šimková et al., 2006; Šimková and Morand, 2008; Mendlová and Šimková, 2014; Grégoir et al.,

70 2015), and to explore the extent of correlation between phenotype variation in attachment organs

71 and factors such as phylogeny, host specificity and geographical location (Vignon et al., 2011).

72 Morphometric variation in anatomical structures of interest can be studied using two

73 approaches. Traditional morphometrics (Reyment et al., 1984; Marcus, 1990) is characterized by

74 the use of lengths of defined positions on anatomical structures of interest (or their ratios) as

75 input data for multivariate statistical analyses. While such variables may measure size adequately,

76 they are generally not effective for capturing shape information present in the geometry of a set

77 of defined points of an object (Rohlf and Marcus, 1993). A large proportion of biological

78 variation due to shape differences is therefore missed when an analysis uses only information

79 from variation in length variables.

80 With the development of geometric morphometrics over the past three decades,

81 researchers now have, at their disposal, a powerful method for extracting, visualizing and

82 combining shape data with other data types such as molecular phylogenies to attain an integrative

83 evolutionary analysis (Rohlf and Marcus, 1993; Adams et al., 2004; Adams et al., 2013).

84 Digitization of the anatomical structure of interest provides the key to the acquisition and use of a

85 new type of data - landmark coordinates, from which shape information can be effectively 
86 extracted, and then analyzed, using new tools such as Procrustes superimposition, thin plate

87 splines, relative warp analysis and elliptic Fourier analysis. Geometric morphometrics is now

88 commonly used in systematics and evolutionary biology research where analysis of shape can be

89 expected to provide new insights to complement traditional morphometric, phylogenetic or

90 biogeographic analyses. A cursory search in major biological journal databases for recent

91 publications having "geometric morphometrics" in their keywords revealed that geometric

92 morphometrics is widely used to study various biological aspects, in diverse phyla, such as fish

93 taxonomy (Sidlauskas et al., 2011), plant taxonomy (Conesa et al., 2012), gastropod shell shape

94 variation (Smith and Hendricks, 2013; Cruz et al., 2012), morphological adaptation in birds

95 (Sievwright and MacLeod, 2012), fly wing evolution (Pepinelli et al., 2013), turtle neck shape

96 evolution (Werneburg et al., 2015), beetle speciation (Pizzo et al., 2013) and species boundary

97 problems in butterflies (Barão et al., 2014). Because of the inherently digital nature of geometric

98 morphometric data, its increasing prominence in morphological studies accentuates the role of

99 informatics in modern taxonomy (Wheeler, 2007).

100 In morphological analyses of monogeneans, taxonomists often prioritize prominent

101 sclerotized parts such as the copulatory organ, because qualitative variation in the latter is

102 frequently sharp and easy to describe. Nonetheless, morphometric variation in all sclerotized

103 parts of monogeneans has been studied for a long time from the perspective of systematics (e.g.

104 Shinn et al., 2001) and evolutionary ecology (e.g. Poisot and Desdevises, 2010; Mendlová and

105 Šimková, 2014). Hard parts such as the anchors are ideal for geometric morphometric analysis

106 because they are not easily deformed by compression when mounted onto slides (Lim and

107 Gibson, 2009). Anchor shape and size are taxonomically informative. Typically, size information

is captured quantitatively in the form of distances between two defined_-points on the anchors, and shape information is captured qualitatively in the form of character states. The analysis of 
111 traditional morphometrics (e.g. Mariniello et al., 2004; Shinn et al., 2004; Tan et al., 2010; Hahn

112 et al., 2011; Soo and Lim, 2012). To date, there are only few examples (Vignon and Sasal, 2010;

113 Vignon, 2011; Vignon et al., 2011; Rodríguez-González et al., 2015a) of applying geometric

114 morphometrics to analyze monogenean anchor shape variation to overcome the limitations in

115 data resolution inherent in standard morphometric and qualitative analyses. The paucity of

116 geometric morphometric studies, however, belies the importance of this approach in uncovering

117 intraspecific shape variation in anchors that can be invaluable for species delimitation,

118 particularly in resolving synonymyiess (e.g. Pérez Ben et al., 2014), as well as for testing

119 hypotheses of morphological integration (Olson and Miller, 1958) and evaluating levels of 120 phenotypic plasticity (Pfennig et al., 2010).

As anchors serve a functional purpose, a priori, it is unclear whether phenotypic similarity

122 of anchors among species is an outcome of adaptive processes related to the ecology or the morphology of their fish host, or simply a reflection of their phylogenetic constraint (Morand et al., 2002). If the presence of phylogenetic signal in anchors can be statistically established, evolutionary analysis of shape and size change can then be used to elucidate trends in particular clades. The results are expected to be useful for guiding the selection of appropriate anchor morphometric variables for conversion into morphological characters that have lower levels of homoplasy, thus overcoming the problem of unnecessary homoplasy of a morphological character arising from poor quality and insufficient number of character states (Perkins et al., 2009).

In this paper, we developed an integrative analysis that uses data from anchor morphometry and morphology, as well as DNA sequences, that allows the investigation of broad aspects in the systematic biology of monogeneans, such as species discrimination, evolutionary

134 ecology, phylogenetic signal, and morphological integration. For illustration, we used data 
136

137

138

139

140

141

Ancyrocephalidae) genus, a particularly speciose genus with 63 species known to date (Table S1). Thus, our study covered all the Ligophorus species (approximately $20 \%$ of global

Ligophorus diversity) currently found on two mullet host species in Southeast Asia. Mullets are eaten by people in this region.

\section{MATERIALS AND METHODS}

Samples

We obtained 530 specimens (metadata in Table S2; sample sizes in Table S3) belonging to 13 Ligophorus species (Soo and Lim, 2012; Soo and Lim, 2015; Soo et al., 2015) from two species of adult fish-mullet host: Liza subviridis Valenciennes, $1836(\mathrm{n}=52)$ and Moolgarda buchanani Bleeker, $1853(\mathrm{n}=29)$ from several locations in tropical Western Peninsular Malaysia (Fig. S1).--The specimens have been deposited in the Museum of Zoology at University of Malaya (508 specimens), the Natural History Museum, London (14 specimens), and the Lee $\underline{\text { Kong Chian Natural History Museum, Singapore (6 specimens). }}$

From 2009 to 2014, Liza subviridis was collected off Carey Island in Selangor (2 $52^{\prime}$ N, $\left.101^{\circ} 22^{\prime} \mathrm{E}\right)$, and M. buchanani off Langkawi Island in Kedah $\left(6^{\circ} 21^{\prime} \mathrm{N}, 99^{\circ} 48^{\prime} \mathrm{E}\right)$ and the sea off Johor $\left(1^{\circ} 20^{\prime} \mathrm{N}, 103^{\circ} 32^{\prime} \mathrm{E}\right)$. The fish were obtained from local fishermen in the vicinity of the sampling locations, dead at the time of purchase. - Seven of the 13 species: L. bantingensis Soo \& Lim, 2012, L. careyensis Soo \& Lim, 2012, L. chelatus Soo \& Lim, 2012, L. funnelus Soo \& Lim, 2012, L. navjotsodhii Soo \& Lim, 2012, L. parvicopulatrix Soo \& Lim, 2012 and L. belanaki Soo \& Lim, 2015 were found on Liza subviridis; the remainder: L. fenestrum Soo \& Lim, 2012, L. kedahensis Soo \& Lim, 2012, L. kederai Soo \& Lim, 2015, L. grandis Soo, Tan \& Lim, 2015, L. johorensis Soo, Tan \& Lim, 2015, and L. liewi Soo, Tan \& Lim, 2015, were found on M. buchanani. For all 81 fish examined, the Ligophorus species found in M. buchanani were never observed in Liza subviridis, and vice versa. 

monogeneans were put onto a clean slide with a drop of water, and then covered with a coverslip. Specimens were initially mounted in modified ammonium picrate glycerine, and subsequently converted into unstained, permanent mounts in Canada balsam. The opisthaptorial sclerotized

164 hard parts of Ligophorus consist of a pair (left and right) of dorsal and ventral anchors, bars, and marginal hooks. Digital images of these hard parts were taken from labelled mounted slides using a light microscope with Leica digital camera (DFC 320) connected to the QWin plus image analysis software (Leica Microsystems, Germany) under 40x magnification, saved as jpeg files 168 and organized into folders. Three species

(L. fenestrum, L. liewi and L. kederai) showed a probable fixed character state - that of the presence of fenestrated structures on anchors of all examined specimens, since no variation in this

171 character state was observed in all examined specimens of these three species.

\section{Data Acquisition}

173 Where possible, we measured a specimen's body length from its anterior to posterior end, 174 and body width at the midpoint of its body. To obtain landmark coordinate data from the anchors, 175 we used TPSDIG2 (Rohlf, 2013; Rohlf, 2015). Eleven landmarks (LM; six Type I, five Type III) 176 were placed sequentially on the right and left ventral and dorsal anchors of each specimen (Fig. 177 1). 
a)

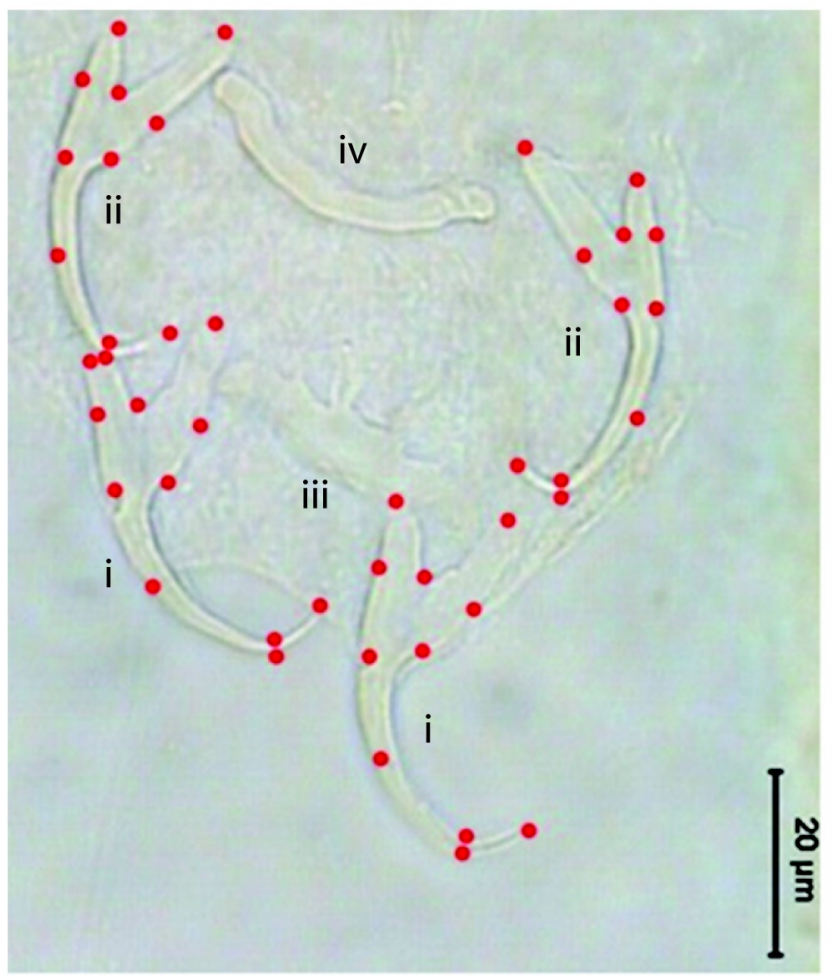

b)

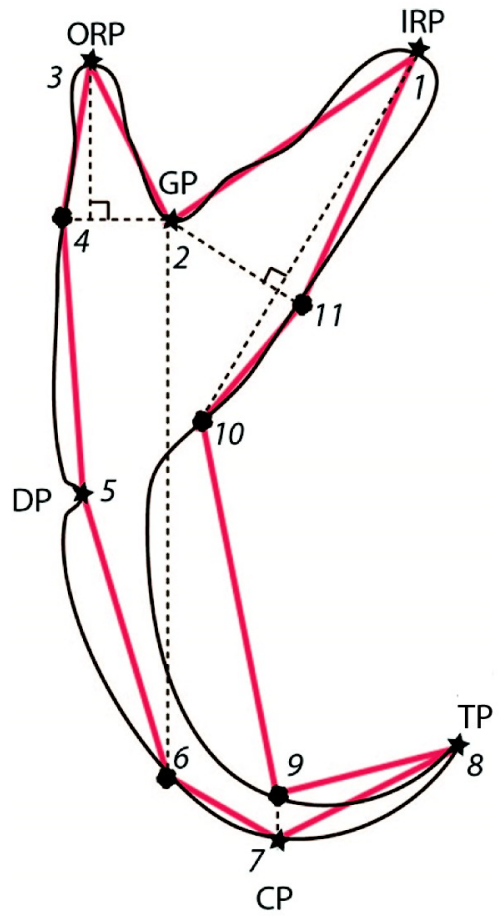

The set of all 11 landmark coordinates makes up a specimen's landmark configuration.

180 Six of the landmarks are Type I (LM1,LM2,LM3,LM5,LM7,LM8), while the remainder root point and the outer root point, respectively. Sandwiched between them is LM2, the groove point. LM5 is the dent point, while LM7 is the curve point. The tip point is represented by LM8.

The semi-landmarks were defined relative to Type I landmarks. The horizontal (towards the outer root point) and vertical projections (towards the curve point) from LM2 intersect with the anchor outline to give LM4 and LM6, respectively. LM9 and LM10 are the intersection points between the vertical projection from LM7 and LM1 with anchor outline, respectively. The projection from 
compartment, and the set LM5 to LM10 to represent the point compartment. For geometric

191 morphometric analysis, semi-landmarks were not specially treated (e.g. employing sliding

192 landmark analysis), following MacLeod (2013) that such treatments may introduce distortions to

193 the original geometrical relationship that lead to complicated interpretations of the result. The

194 anchor images and their corresponding landmark coordinate data are given in Supplemental

195 Material D1 and D2, respectively.

\section{Data Processing and Analysis Tools}

We created a new R (Version 3.2.1; R Core Team, 2015) package called monogeneaGM (Khang, 2015) to process raw landmark coordinate data and integrate new methodological developments in the current study with numerous data processing and analysis tools in $\mathrm{R}$ packages, such as geomorph (Adams and Otarola-Castillo, 2014), phytools (Revell, 2012), circular (Agostinelli and Lund, 2013), gplots (Warnes et al., 2014), ape (Paradis et al. 2004), rgl (Adler et al., 2014) and cluster (Maechler et al., 2015).

The monogeneaGM package contains a suite of $\mathrm{R}$ functions for three primary analyses: anchor landmark coordinate data quality control, visual checks and Generalized Procrustes Analysis; hierarchical clustering and principal component analysis; and analysis of anchor shape change using directional statistics. Some of the functions are suitable for general use. For example, the phylomorphospace visualization functions - tpColorPlot $2 \mathrm{~d}$ for twodimensional data, and tpColorPlot $3 \mathrm{~d}$ for three-dimensional data, can take in two additional arguments: a color transparency control, which is useful for improving graphical presentation when there are substantial overlaps of data points, and an option to superimpose a user-supplied phylogeny onto defined centroids in the scatter plot, if this information is available.

\section{Data Quality Control}


214 would contain substantial amount of non-biological shape variation caused by incongruent image 215 and object planes (Arnqvist and Mårtensson, 1998). The inclusion of these poor quality data in 216 downstream analyses is undesirable, as they introduce noise into an analysis that can potentially 217 complicate the interpretation of results. To mitigate this problem, we developed a quality control 218 procedure to filter out poor quality images. In this procedure, we first computed all pairwise

219 Euclidean distances between landmarks for the left and right forms of dorsal and ventral anchors.

220 If both left and right forms have congruent image and object planes, then by symmetry, their

221 residual - the difference of their pairwise Euclidean distances for each landmark $(M)$, should be 222 close to zero, thus yielding a small sum of squared residuals $\left(M^{2}\right)$. Moreover, we expect $M$ to be randomly distributed with zero mean across all average pairwise Euclidean distances $(A)$ between the left and right forms. The slope of the regression equation of $M$ against $A(b)$ allows us to measure how well this expectation is satisfied. To be comparable with the sum of squared residuals, we squared the estimated regression slope $\left(b^{2}\right)$, and then scaled it to be on the same order of magnitude as $M^{2}$. Thus, a good quality specimen would have small sum of $M^{2}$ and $b^{2}$, 228 and vice versa. We defined the quality score $Q$, as

$$
Q=100 \times 10^{\frac{-\sqrt{M^{2}+b^{2}}}{10}}
$$

230 The magnitude of this measure is straightforward to interpret - it is high (maximum 100) for 231 good quality specimens and low (minimum 0) for poor ones. Figure 2 shows examples of poor 232 and good quality specimens together with their Tukey Mean-Difference (TMD) plots, 233 respectively. Specimens with $Q$ of 10 or more $(n=437$; Table S3 and Fig. S2) were used for 234 subsequent analyses. 
a)

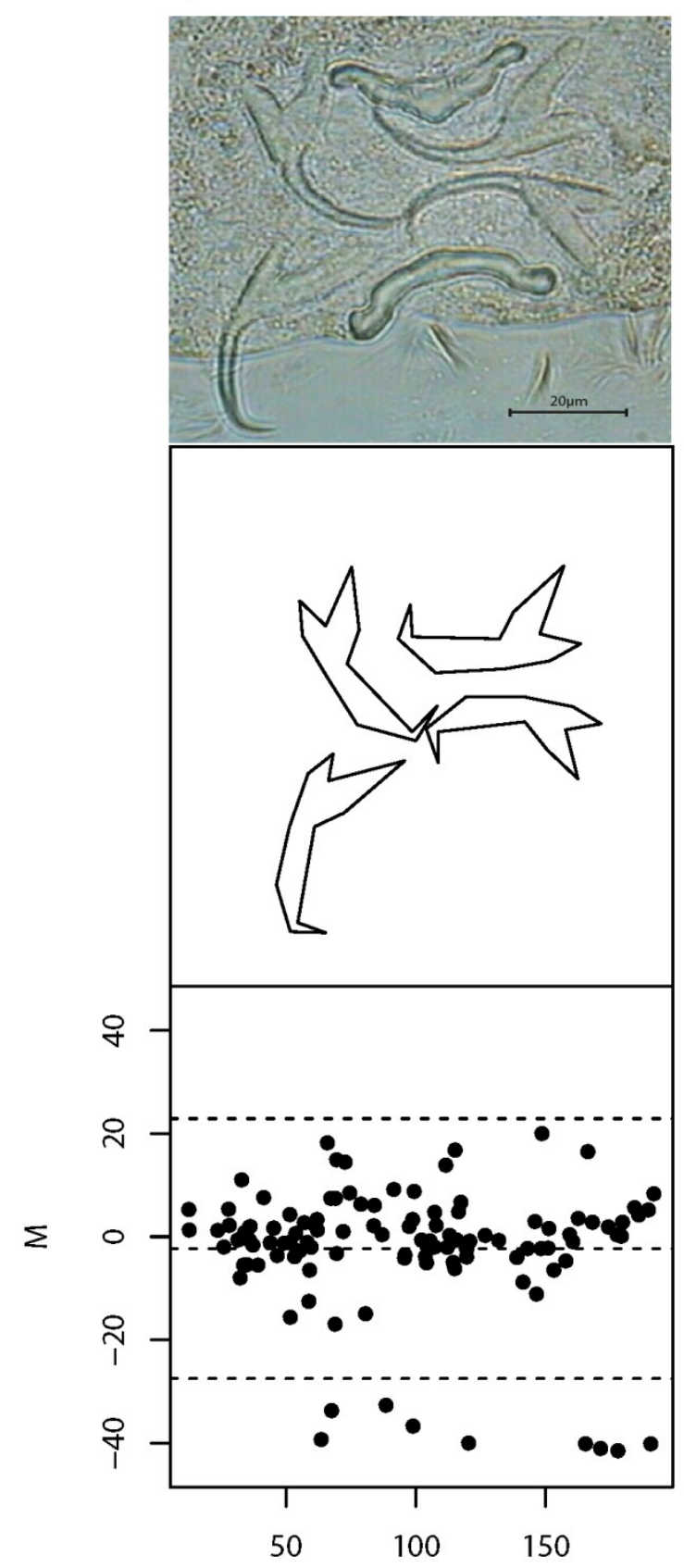

A b)

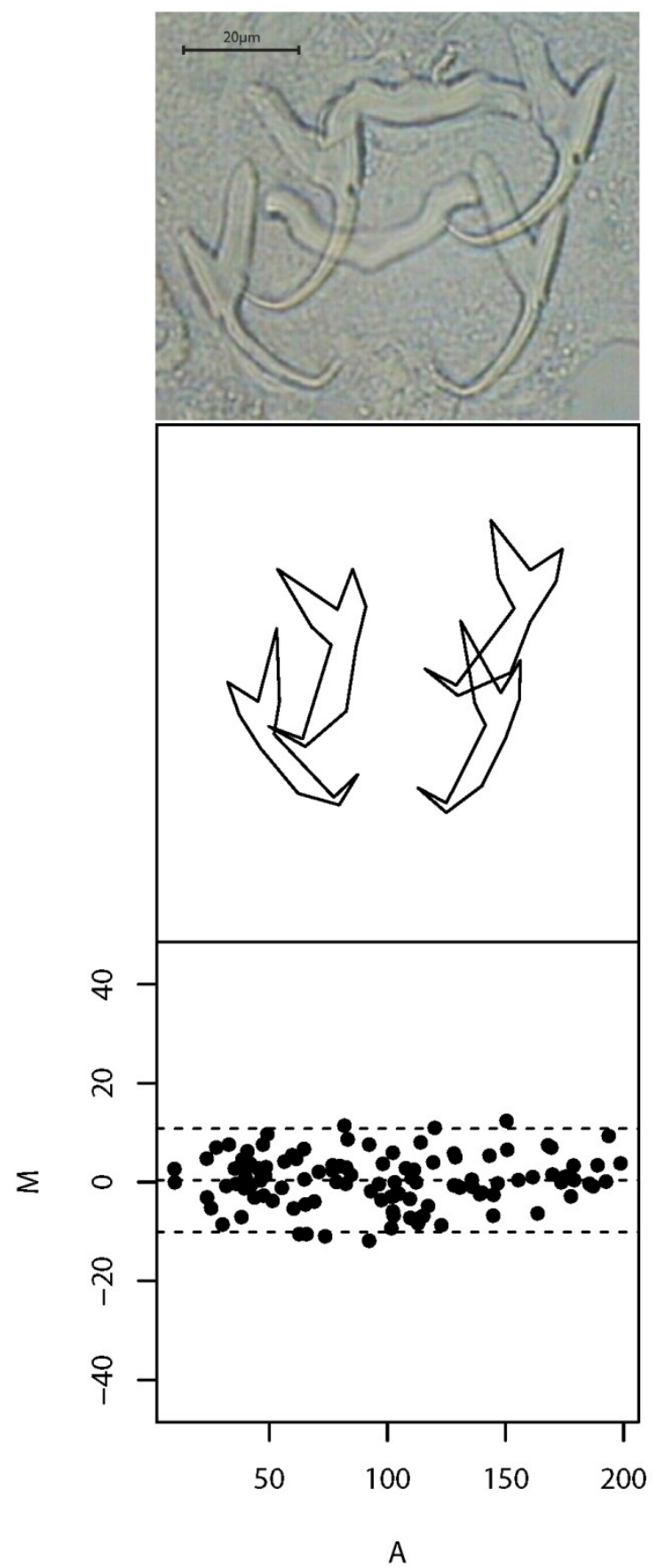

We used a subset $(n=96)$ of the total specimens with quality score above $10(n=437)$ and measured the physical distances from LM1 to LM3 and from LM1 to LM5 in these samples 
240 physical distances against the computed pairwise Euclidean distances to determine the linear

241 equation for converting arbitrary distance units into their physical units (in $\mu \mathrm{m})$. Thus, all

242 pairwise Euclidean distances computed from raw landmark coordinates could be converted to

243 physical distances by multiplication with a factor of 0.2 followed by addition of 0.9 (Fig. S3).

244 Geometric Morphometric Analysis

245 For each species, we performed Generalized Procrustes Analysis (GPA; Gower, 1975;

246 Rohlf and Slice, 1990) to align the sample landmark configurations for both ventral and dorsal

247 anchors, using the gpagen function in the geomorph package (Version 2.1.1; Adams and

248 Otarola-Castillo, 2014). The resulting GPA coordinates of the left and right forms were then

249 averaged. GPA removes the effects of translation, rotation and scaling so that the resulting

250 landmark configurations have minimum sum of squared distances with respect to the mean

251 landmark configuration (Adams et al., 2004). Nevertheless, even after GPA, comparison of

252 anchor shape variation can still be potentially confounded by the presence of non-biological

253 variation in the landmark configuration. Specifically, if many samples of a species have anchors

254 lying in one particular position, it would not be clear whether variation between its members'

255 mean GPA landmark configuration and those of other species constitutes genuine biological

256 variation or mathematical artifact. Typical application of geometric morphometrics in non-

257 microscopic objects (fly wings, skulls, etc.) does not usually suffer from this problem, since

258 specimens from species to be compared can be manipulated into standardized positions before

259 imaging.

260 To ensure that the landmark configurations of all 13 species were comparable, we

261 determined the angular deviation of LM7 from the $x=0$ line, and rotated all landmark coordinates

262 by this amount with the origin as pivot. This has the effect of creating standardized landmark

263 configurations for specimens across all species since the $\mathrm{x}$-coordinate of LM7 is always zero after 
264 adjustment. The GPA coordinate data of all specimens thus obtained were subjected to another

265 iteration of GPA to produce the final shape alignment, which was organized into a data matrix

266 with rows representing specimens and columns representing the 44 GPA landmark coordinates.

267 Molecular Phylogenetic Analysis

268 We used DNA sequence data from three nuclear markers: 28S rRNA, 18S rRNA and ITS1

269 from the 13 Ligophorus species to infer their phylogenetic tree. These markers are a mainstay of

270 parasitic platyhelminth molecular phylogenetics (Littlewood, 2008), and the combination of fast

271 (ITS-1) and slow (28S rRNA, 18S rRNA) evolving sequences should provide sufficient

272 resolution for the inferred phylogenetic tree (Lockyer et al., 2003; Blair, 2006; Waeschenbach et

273 al., 2007), allowing the relationship of even closely related monogenean species to be resolved

274 (e.g. in Gyrodactylus (Gilmore et al., 2012)). Ideally, the inclusion of mitochondrial markers such

275 as cytochrome oxidase I (COI) would provide a more representative sampling of the genome, and

276 hence, a more reliable phylogeny. However, the absence of mitochondrial data may not impact

277 the quality of the inferred phylogeny, since, for monogeneans, it has been shown in Diplectanidae

278 and Gyrodactylus that both rRNA and mitochondrial markers are effective for species

279 identification (Vanhove et al., 2013). Regardless of whether nuclear or mitochondrial genes are

280 used, it is important to keep in mind that gene trees do not always reflect the species tree

281 (Maddison, 1997).

282 Partial 28S rRNA ( 800bp) and ITS1 ( 750bp) sequence data were obtained from Soo et

283 al. (2015), whereas 18S rRNA sequence data were generated in the present study. Briefly, the

284 Ligophorus specimens were removed from host gills, identified morphologically and then

285 preserved in 75\% ethanol. Genomic DNA was extracted from samples using DNAEasy extraction

286 kit (QIAGEN, Hilden, Germany). About $5 \mu 1$ of the extracted DNA was used as template in the

287 PCR reaction to amplify the partial $18 \mathrm{~S}$ rRNA sequence using two primers: WormA (5'- 
GCGAATGGCTCATTAAATCAG-3') (Littlewood and Olson, 2001) and new930F (5'-

CCTATTCCATTATTCCATGC-3') (modified from Littlewood and Olson, 2001). The PCR

reaction $(50 \mu \mathrm{l})$ was carried out in a solution containing $1.5 \mathrm{mM} \mathrm{MgCl}$, PCR buffer (Fermentas),

$200 \mu \mathrm{M}$ of each deoxyribonucleotide triphosphate, $1.0 \mu \mathrm{M}$ of each PCR primer and $1 \mathrm{U}$ of Taq polymerase (Fermentas), in a thermocycler (Eppendorf Mastercycler) using the following conditions: initial denaturation at $95^{\circ} \mathrm{C}$ for 4 minutes, followed by 35 cycles of $95^{\circ} \mathrm{C}, 52^{\circ} \mathrm{C}$ and $72^{\circ} \mathrm{C}$ for one minute each, with final extension at $72^{\circ} \mathrm{C}$ for 10 minutes. An aliquot $(10 \mu 1)$ from the amplicons were electrophoresed in 1.3\% agarose gel, stained with ethidium bromide and

296 viewed under an ultraviolet illuminator. The remaining $40 \mu 1$ of each amplicon was purified using 297 a DNA purification kit (QIAGEN, Hilden, Germany) and subjected to automated DNA sequencing (ABI 3730 DNA Sequencer, First Base Laboratories, Kuala Lumpur) using the same primers used for PCR amplification. Approximately $750 \mathrm{bp}$ of the $18 \mathrm{~S}$ rRNA sequence were amplified and sequenced for the 13 Ligophorus species (Table 1).

\begin{tabular}{llllll}
\hline Ligophorus & Host species & $\begin{array}{l}\text { Locality } \\
\text { species }\end{array}$ & & \multicolumn{3}{c}{ GenBank Accession no. } \\
& & & & & \\
\cline { 3 - 5 } & & & 28S rRNA & 18S rRNA & ITS1 \\
\hline
\end{tabular}




\begin{tabular}{|c|c|c|c|c|c|}
\hline L. bantingensis & $\begin{array}{l}\text { Liza } \\
\text { subviridis }\end{array}$ & $\begin{array}{l}\text { Carey Island, } \\
\text { Selangor }\end{array}$ & KM221909 & KM221934* & KM221922 \\
\hline L. belanaki & & & KM221910 & KM221935* & KM221923 \\
\hline L. careyensis & & & KM221911 & KM221936* & KM221924 \\
\hline L. chelatus & & & KM221912 & KM221937* & KM221925 \\
\hline L. funnelus & & & KM221914 & KM262663* & KM262662 \\
\hline L. navjotsodhii & & & KM221920 & KM221944* & KM221932 \\
\hline L. parvicopulatrix & & & KM221921 & KM221945* & KM221933 \\
\hline L. fenestrum & $\begin{array}{l}\text { Moolgarda } \\
\text { buchanani }\end{array}$ & $\begin{array}{l}\text { Langkawi } \\
\text { Island, } \\
\text { Kedah }\end{array}$ & KM221913 & KM221938* & KM22 \\
\hline L. kedahensis & & & KM221917 & KM221941* & KM221929 \\
\hline L. kederai & & & KM221918 & KM221942* & KM221930 \\
\hline L. grandis & & $\begin{array}{l}\text { Straits of } \\
\text { Johor }\end{array}$ & KM221915 & KM221939* & KM221927 \\
\hline L. johorensis & & & KM221916 & KM221940* & KM221928 \\
\hline L. liewi & & & KM221919 & KM221943* & KM221931 \\
\hline
\end{tabular}

\section{TABLE 1.}

303 (Version 7 at http://mafft.cbrc.jp/alignment/server/; Katoh and Standley, 2013). The alignment

304 parameters used were the Q-INS-i iterative refinement method, the $1 \mathrm{PAM} / \kappa=2$ nucleotide scoring matrix with a gap opening penalty of 1.53 . We then concatenated multiple sequence alignments of the three nuclear markers (28S rRNA-ITS1-18S rRNA). The resulting supermatrix was used as input in IQ-TREE (Nguyen et al. 2015) to construct the maximum likelihood (ML) phylogenetic tree (Felsenstein, 1981; Felsenstein, 2003). IQ-TREE is a state-of-the-art ML tree construction pipeline that integrates DNA model selection, maximum likelihood tree search, and rapid bootstrap analysis (Minh et al., 2013). For model selection, we used the Bayesian Information

311 Criteria, and did not consider "G+I" models, following Yang (2006) that modelling proportion of 312 invariable sites in the presence of gamma rate variation across sites creates model identifiability 313 issues. MEGA (Version 6; Tamura et al., 2013) was used to edit the resulting phylogenetic tree. 314 We annotated the tree with the morphology of anchors, bars and male copulatory organ to allow 315 visual assessment of overall phylogenetic and phenotypic correlation. 
316

317

318

319

320

321

322

324

325

326

327

328

329

330

331

332

333

334

335

336

337

338

339

\section{Species Discrimination}

Discriminating a monogenean species is a complex art that involves the comparison of qualitative features of numerous anatomical structures: the male copulatory organ, female reproductive organ, anchors, bars and marginal hooks. Among the sclerotized hard parts, multivariate morphometric analyses of shape and size variables of suitable anatomical structures provide a quantitative means for species discrimination, which is invaluable for complementing the results from qualitative morphological analyses.

To visualize species clustering in low dimension morphospace, we applied Principal Component Analysis (PCA) separately for the ventral and dorsal anchors using their GPA coordinate data. The trade-off between loss of information through dimensional reduction and gain of interpretation via visualization in PCA can, however, make it difficult to judge how well members of the same species cluster together in the PCA scatter plots, especially when there are overlaps between different species clusters. To overcome this problem, we complemented PCA results with the cluster heat map (Wilkinson and Friendly, 2008), a powerful method for organizing high-dimensional multivariate data that allows visual detection of patterns of variation. The cluster heat map first maps numerical information in the cells of the input data matrix to corresponding color codes. Then, a hierarchical clustering algorithm is applied to cluster the samples by similarity, in such a way that within cluster variation is always smaller than between cluster variation. For the current analysis, we estimated similarity between each pair of samples using the Manhattan distance metric. The resulting distance matrix was then used as input for hierarchical clustering of samples using the Ward algorithm.

To assess the impact of applying the quality control procedure, we compared cluster heat maps generated using all samples, and using only samples that passed data quality control. Heat map construction was done using the heatmap. 2 function in the gplots package (Version 
340 2.13.0; Warnes et al., 2014). We found the simple heat map a good alternative to inspection of the

341 PC loadings table when trying to interpret the first few PC axes biologically.

342 Testing for the Presence of Phylogenetic Signal in Anchor Shape

343 Species with different shapes are localized in particular regions of the morphospace.

344 When a phylogeny is superimposed onto this morphospace, a phylomorphospace is induced, and

345 it becomes possible to evaluate whether common descent or convergent evolution is likely to

346 have shaped phenotypic similarity (Klingenberg and Ekau, 1996; Sidlauskas, 2008; Revell,

347 2014). If anchor shape contains substantial phylogenetic signal, then we expect the phylogeny to

348 have non-random branching patterns in phylomorphospace. Graphically, we may visualize the

349 latter by superimposing the molecular phylogeny of Ligophorus on the PCA plots of the first

350 three principal components for the ventral and dorsal anchors. Estimation of ancestral node

351 positions in the phylomorphospace was done using the maximum likelihood method as

352 implemented in the fastAnc function of the phytools package (Version 0.4-21; Revell,

353 2012).

354 We formally tested the presence of phylogenetic signal in anchor shape by applying

355 Adams's multivariate $\mathrm{K}_{\text {mult }}$ test (Adams, 2014a), implemented using the physignal function

356 (10 000 iterations) in the geomorph package (Version 2.1.1; Adams and Otarola-Castillo, 2013).

357 The $\mathrm{K}_{\text {mult }}$ statistic is a multivariate generalization of Blomberg's K statistic (Blomberg et al.,

358 2003). The phenotype (a continuous trait) interest in the lineages of a given phylogeny is

359 assumed to evolve in phylomorphospace according to Brownian motion. In the absence of

360 phylogenetic signal, $K_{\text {mult }}=0$ (i.e. phenotypic variation is independent of lineages). At $K_{\text {mult }}=1$,

361 phenotypic variation between taxa in the same lineage conforms to expectation under Brownian

362 motion evolution. Values of $\mathrm{K}_{\text {mult }}<1$ correspond to phenotypic variation that is larger than 
363

364

365

366

expected between taxa of the same lineage, and values of $\mathrm{K}_{\text {mult }}>1$ correspond to phenotypic variation that is smaller than expected between taxa of the same lineage (Adams, 2014a).

Statistical significance is determined via a permutation procedure under assumption of phylogenetic signal absence.

\section{Analysis of Anchor Shape and Size Evolution}

In studying anchor shape and size evolution, we were primarily concerned with trends occurring in different clades of the phylogeny of the 13 Ligophorus species. To control for the effect of body size in subsequent phylogenetic regression analysis of anchor shape against anchor size, it was necessary to first test for collinearity of body size and anchor size (Mundry, 2014).

Since body size was prone to distortion during fixation, we used the median of body length and body width of each species to reduce the impact of outliers. For analysis, the logarithm (base 10) of the product of median body length and median body width was used.

The GPA landmark coordinates of the ancestral anchor were estimated using the maximum likelihood method as implemented in fastAnc function from the phytools package (Version 0.4-21; Revell, 2012). Anchor shape change associated with a clade is statistically supported if mean directional change deviates significantly from uniformity in a set of landmarks. We visualized directional deviation in the 11 landmarks of both ventral and dorsal anchors using circular plots (Agostinelli and Lund, 2013; implemented in the circular package, Version 0.4-7). We then performed Rayleigh's test (Batschelet, 1981) to test for evidence against directional uniformity in each landmark. The strength of statistical evidence against mean directional uniformity in each landmark was assessed using p-value. Wireframelollipop plots (Klingenberg, 2013) were used to graphically summarize the mean change in direction and mean magnitude of landmark displacement from root ancestor landmark configuration. 
For investigating trends in anchor size evolution, we first computed all possible pairwise

388 Euclidean distances between the raw landmarks in each sample. Each dorsal and ventral anchor 389 has 11 landmarks, thus generating 55 possible pairwise Euclidean distances which we used as 390 size variables. When the loadings and variables of the first principal component (PC1) have the 391 same sign, PC1 can be interpreted naturally as a measure of size (Jolliffe, 2002). Subsequently, 392 we performed continuous character mapping (implemented using contMap function in the 393 phytools package) of mean PC1 of the size variables of each species for ventral and dorsal 394 anchors onto the phylogeny of 13 Ligophorus species to assess clade-specific patterns of anchor 395 size evolution. The Adams-Collyer phylogenetic regression for shape response variable (Adams, 396 2014b; Collyer et al. 2015; implemented in the geomorph package, Version 2.1.1) was used to 397 formally test evolutionary correlation between anchor shape and two covariates: the logarithm of 398 body size and the logarithm of anchor size. The interaction between the two covariates was 399 incorporated into the phylogenetic regression model if covariate collinearity could be ruled out 400 using the Ho-Ané phylogenetic regression (Ho and Ané, 2014; implemented in the phylolm 401 package, Version 2.2) under a Brownian motion model for the phylogenetic covariance matrix. 402 For both regression analyses, p-values were computed via a resampling procedure with 10000 403 iterations. 
408 similar microhabitats); conversely, when parasites have dissimilar attachment organs (thus

409 occupying different microhabitats), the morphology of their copulatory organs would not show

410 important differences, since the lack of proximity puts less evolutionary pressure on the parasites

411 to evolve morphologically different copulatory organ. Qualitative evidence with limited number

412 of congeneric species (Lambert and Maillard, 1975; Roubal, 1981; Rohde et al., 1994) supported

413 the hypothesis's feasibility, as well as later studies with more species (Šimková and Morand,

414 2008, 2015). Quantitative evaluations using larger species assemblage that relied on traditional

415 morphometric data are available, but the interpretation of their results in support of the hypothesis

416 was obscured by either the problem of using inflated degrees of freedom in regression analysis

417 (e.g. Šimková et al., 2002) or failure to control for the effect of phylogeny (e.g. Jarkovský et al.,

418 2004). With the development of new tools for geometric morphometric and phylogenetic

419 comparative methods, we are in a position to retest the Rohde-Hobbs hypothesis. To this end, we 420 compared the size of the male copulatory organ (mean tube length data from Soo and Lim, 2012,

421 2015; Soo et al., 2015) and three of its selected morphological characters (Table 2: position of

422 copulatory organ entrance at the main lobe of accesory piece; accesory piece of male copulatory

423 complex; shape of accesory piece of male copulatory complex) against anchor shape and size

424 variation. Ancestral node positions were estimated as before using the fastAnc function in the 425 phytools package. 


\section{Characters}

Male copulatory organ:

Position of copulatory organ entrance at the main lobe of accessory piece

Accessory piece of male copulatory complex

Shape of accessory piece of male copulatory complex

\section{$\underline{\text { Female reproductive system }}$}

Vaginal canal sclerotization

Distal end of sclerotized vagina

$\underline{\text { Bars }}$

Relative size of ventral and dorsal bar

$\underline{\text { Anchors }}$

Ratio of shaft to point of ventral anchor

Ratio of shaft to point of dorsal anchor

Length of ventral anchor point

\section{Character States}

\begin{tabular}{l} 
Set \\
\hline Included \\
in study
\end{tabular}

(0) proximal; (1) distal; (2) medial

(0) consists of two lobes (main and secondary lobes or proximal and distal ones); (1) consists of one lobe

(0) beak or hook-shaped; (1) claw-shaped pincer-like; (2) crossshaped; (3) funnel-shaped; (4) open, grooved tube (rod-like)

(0) present; (1) absent

(0) funnel-shaped thin-walled; (1) funnel-shaped thick-walled; (2) scyphoid narrow; (3) scyphoid broad; (4) not observed

(0) subequal; (1) dorsal bar longer than ventral one; (2) ventral bar longer than dorsal one; (3) not applicable

(0) less than 1.4 ; (1) $1.4-2.6$; (2) greater than 2.6

(0) less than 1.4 ; (1) $1.4-2.6$; (2) greater than 2.6

(0) 7-12 $\mu \mathrm{m}$; (1) less than $7 \mu \mathrm{m}$

in study$$
\sqrt{ }
$$$$
\sqrt{ }
$$

Set B

Index Included in study

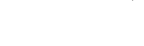

$\sqrt{ }$

$\sqrt{ }$

$\begin{array}{ll}4 & \sqrt{ } \\ 5 & \sqrt{ }\end{array}$

$\begin{array}{llll}\sqrt{ } & 5 & \sqrt{ }\end{array}$

Characters

Character States

\section{Set A}

Included Index Included

in study in study

(0) greater than $11 \mu \mathrm{m}$; (1) 5-11 $\mu \mathrm{m}$; (2) less than $5 \mu \mathrm{m}$

$\mathrm{X}$

$\mathrm{X}$ than point

(0) outer root shorter than point; (1) outer root subequal with point;

(2) outer root longer than point

\section{$\underline{\text { New characters }}$}

Shape of ventral anchor

Shape of dorsal anchor
(0) shaft scimitar-shaped, root U-shaped (1) shaft scimitar-shaped, root V-shaped; (2) shaft sickle-shaped, root U-shaped; (3) shaft sickle-shaped, root V-shaped

(0) shaft scimitar-shaped, asymmetric inner and outer roots; (1) shaft sickle-shaped, symmetric inner and outer roots; (2) shaft sickleshaped, asymmetric inner and outer roots 
Ventral anchor: Length from L1 to L3

Dorsal anchor: Length from L1 to L3

Ventral anchor: Length from L1 to L5

Dorsal anchor: Length from L1 to L5
(0) $15 \mu \mathrm{m}$ or less; (1) greater than $15 \mu \mathrm{m}$

(0) $15 \mu \mathrm{m}$ or less; (1) greater than $15 \mu \mathrm{m}$

(0) Less than $15 \mu \mathrm{m}$; (1) $15 \mu \mathrm{m}-25 \mu \mathrm{m}$; (2) greater than $25 \mu \mathrm{m}$

(0) $15 \mu \mathrm{m}-25 \mu \mathrm{m}$; (1) greater than $25 \mu \mathrm{m}$

$\begin{array}{ccc}\sqrt{ } & 9 & \mathrm{X} \\ \sqrt{ } & 10 & \mathrm{X} \\ \sqrt{ } & 11 & \mathrm{X} \\ \sqrt{ } & 12 & \mathrm{X}\end{array}$

TABLE 2. 
427

428

429

430

431

433

\section{Morphological Integration Analysis}

The roots of the anchor are bases for muscle attachment. Biomechanically, force exerted through the muscles and transmitted to the point compartment controls the anchor's grip strength on the gills. Because of this, we may expect the anchor to function as a single, fully integrated module (Klingenberg, 2008) on a priori grounds. Anchor shape is strongly constrained by either phylogeny or convergent evolution. If the latter's effect was weak, then suitable morphological characters based on variation in anchor shape can be expected to be systematically useful.

To date, only few morphological integration analyses in monogeneans have been done. Using published morphological drawings, Vignon et al. (2011) investigated interspecific modularity of attachment organs (marginal hooks, anchors and bars) in 66 Cichlidogyrus (Monogenea: Ancyrocephalidae) species. More recently, Rodríguez-González et al. (2015a) studied intraspecific morphological integration of the root and the point compartments of anchors in Ligophorus cephali, using the partial least squares method in the context of shape analysis (Rohlf and Corti, 2000). Here, we extended their morphological integration analysis to the interspecific level in Ligophorus. We applied the phylogeny-aware partial least squares method based on the evolutionary covariance matrix (Adams and Felice, 2014) to estimate the extent of morphological integration between the ventral and dorsal anchors, as well as that of the root compartment (L1 to L4 and L11) and the point compartment (L5 to L10) within and between the ventral and dorsal anchors.

\section{New Morphological Characters from Morphometric Variables}

A continuous morphometric variable can be discretized and treated as a morphological character with two or more states for use in a cladistic analysis (Thiele, 1993; Rae, 1998; Wiens, 2001). In doing so, the taxonomist relies on experience and intuition to select promising 
450 morphometric variables out of a potentially large set of candidates. Unfortunately, an objective

451 means to screen the latter is generally lacking. As a result, it is difficult to assess the level of 452 homoplasy present in the taxonomist's candidate characters. Here, we show how comparison of

453 patterns of shape change in different clades leads to the discovery of new morphometric variables

454 for morphological phylogenetic analysis in Ligophorus. A set of 12 morphological characters 455 defined in Sarabeev and Desdevises (2014) that are not invariant for the 13 Ligophorus species

456 (Table 2; see Table S4 for character state matrix) was chosen. We replaced the morphological 457 characters derived from traditional morphometric measurements of anchors with new candidates 458 derived from geometric morphometric analysis to assess their phylogenetic informativeness. To 459 this end, we compared how well-resolved the resulting maximum parsimony trees (using PAUP; 460 Swofford, 2002) were. Tree search (initial tree obtained via stepwise addition) was performed 461 using the heuristic search option. Branch-swapping was done using the tree bisection and 462 reconnection algorithm. Tree reliability was assessed using 1000 bootstrap replicates and 463 branches were collapsed if bootstrap support was below 50\%.

\section{RESULTS}

465 Molecular Phylogeny

466 The GTR + G model was the best DNA substitution model that did not incorporate the 467 proportion of invariable sites. After mid-point rooting, the estimated ML tree (Fig. 3; 10000 468 bootstrap replicates) contained two major clades. One of them consisted of species infecting $M$. 469 buchanani (Clade I), and the other consisted of species infecting Liza subviridis (Clade II).

470 Bootstrap support was high for most internal nodes, except the most recent common ancestor 471 node of L. parvicopulatrix-L. bantingensis, and L. grandis-L. fenestrum (between 50-60\%). 


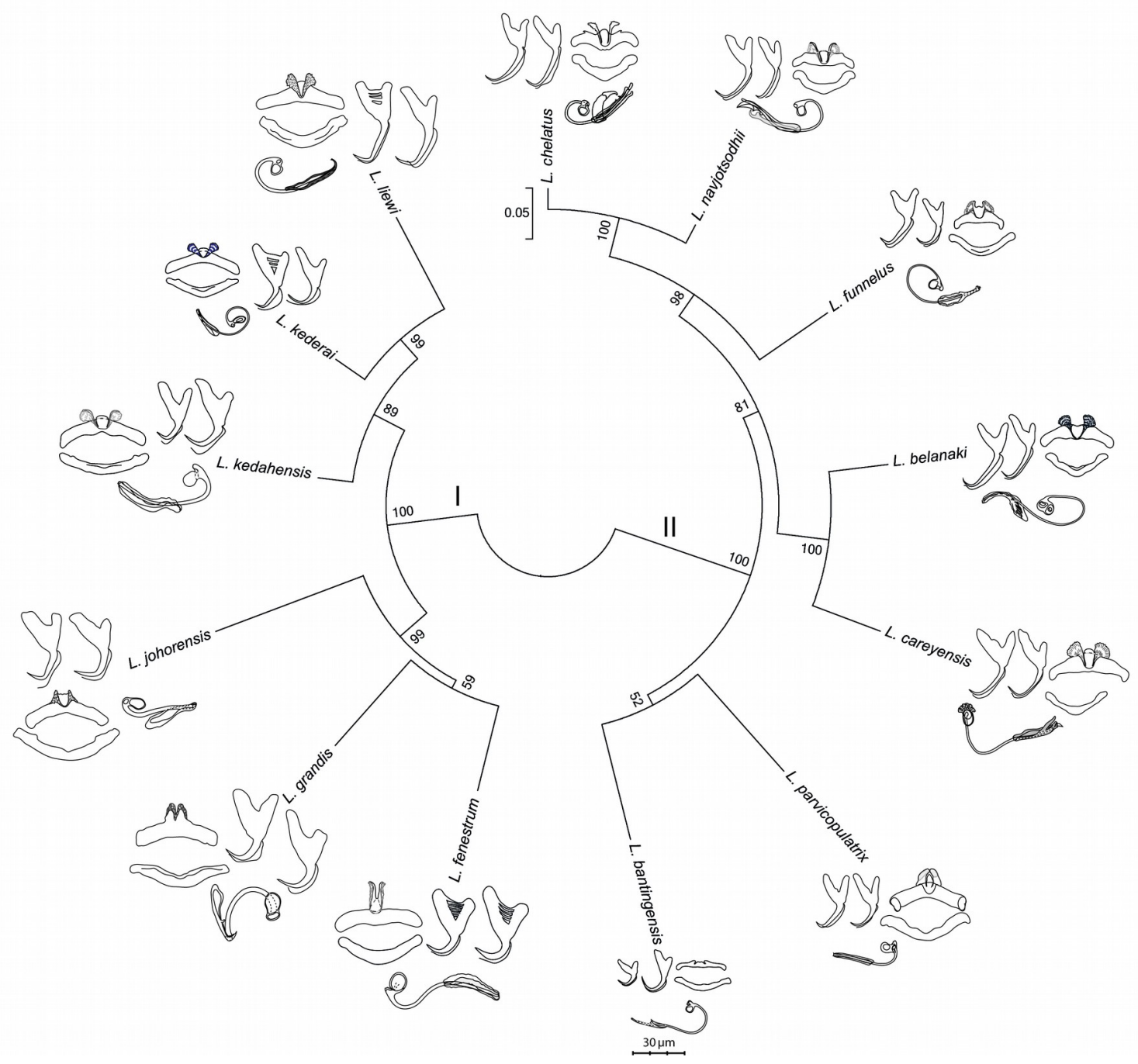

472

FIGURE 3.

473 Morphometry Summary Statistics

474 Table S5 gives summary statistics of anchor size, anchor shape, body size and male 475 copulatory organ size for the 13 Ligophorus species. 
478 anchors are given in Fig. 4 (see Fig. S4 to Fig. S16 for species-specific alignments). Figure 5

479 shows the PCA plots of PC2 against PC1, and PC3 against PC1 for shape variables of ventral and

480 dorsal anchors (See Fig. S17 for a three-dimensional PCA plot). The first three PC accounted for

$48184 \%$ and $79 \%$ of total shape variation in the ventral and dorsal anchors, respectively. To interpret

482 these three PCs, we simultaneously compared the scatter plots of the GPA landmark

483 configurations with the heat map of shape variable loadings (Fig. S18 and S19). Anchors with a

484 sickle-shaped shaft had large positive values of PC1 (e.g. ventral anchors of L. fenestrum, L.

485 grandis, L. kedahensis, L. johorensis), while those with a scimitar-shaped shaft (e.g. ventral

486 anchors of L. chelatus, L. belanaki, L. navjotsodhii) had large negative values in ventral anchors.

487 For ventral anchors, large positive PC2 values were associated with V-shaped root grooves (e.g.

488 L. bantingensis). In contrast, the U-shaped root groove (e.g. L. liewi, L. kederai) was associated

489 with negative PC2 values. For dorsal anchors, PC2 was positive and large for highly symmetric

490 inner and outer roots (e.g. L. parvicopulatrix) and vice versa (L. liewi, L. bantingensis, $L$.

491 grandis). PC3 did not admit a simple geometrical interpretation.

492 The result of Adams's $\mathrm{K}_{\text {mult }}$ test supported the presence of significant phylogenetic signal

493 in anchor shape $(\mathrm{K}=0.948$; $\mathrm{p}$-value $=0.0003)$. Graphically, this is reflected in the PCA plots

494 where both clades show divergent evolutionary trajectories in phylomorphospace (Fig. 5). 
a)

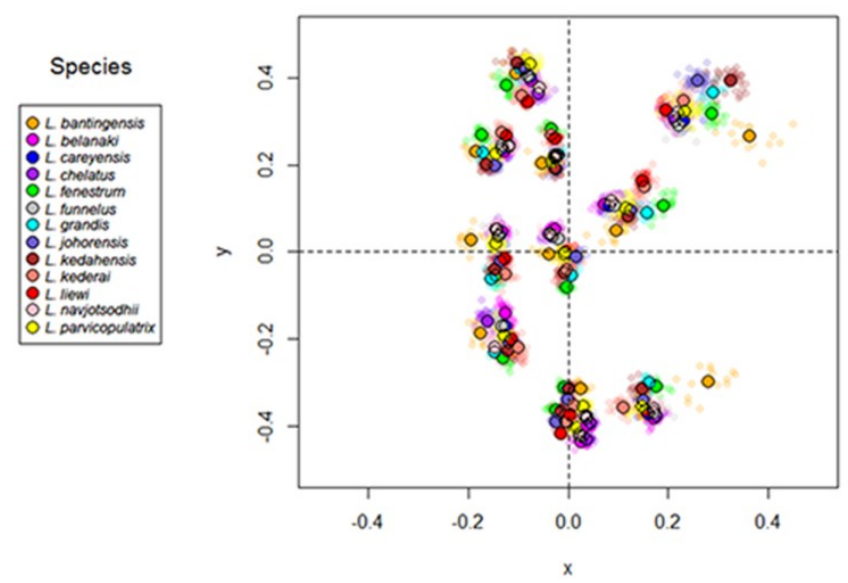

b)

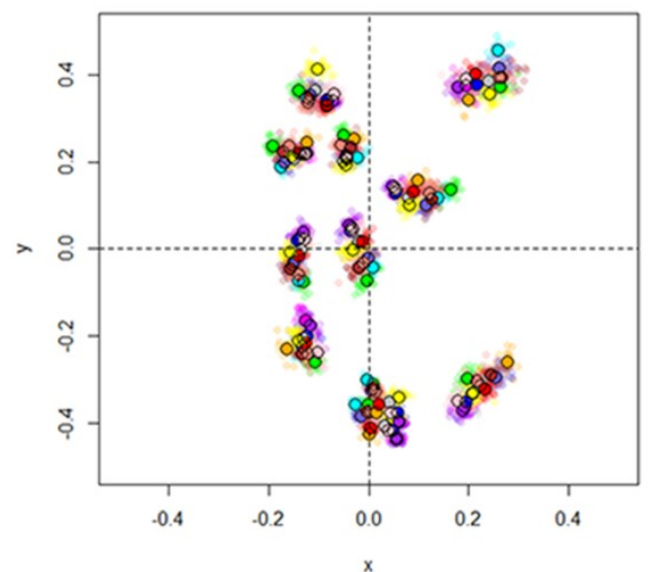


a)

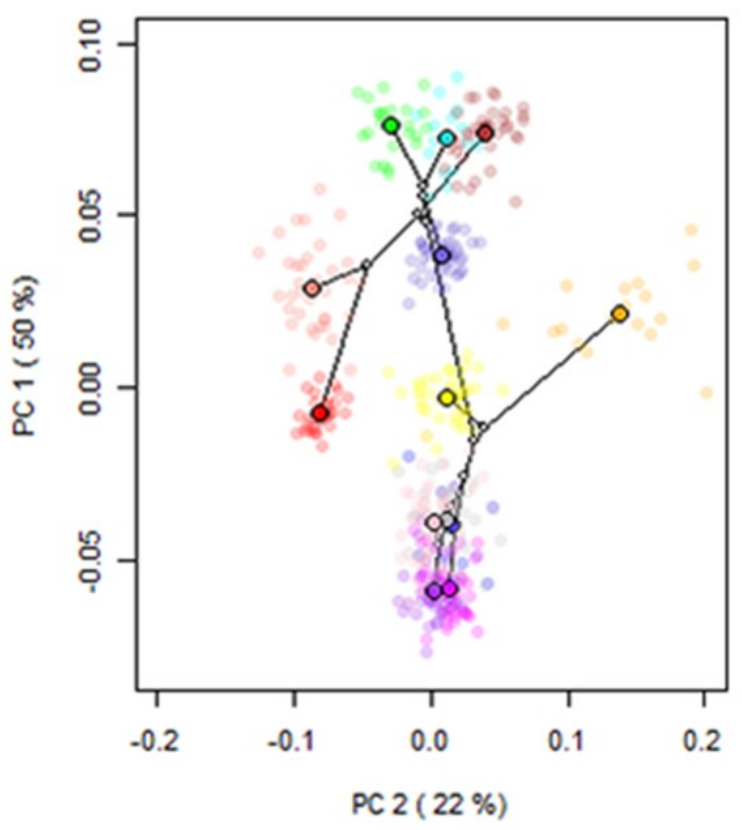

b)

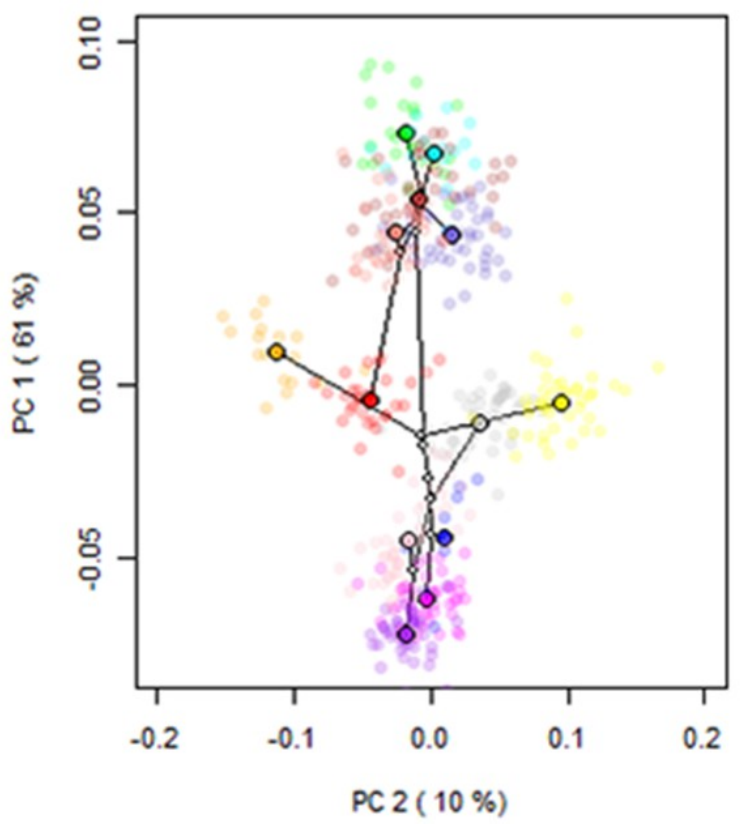

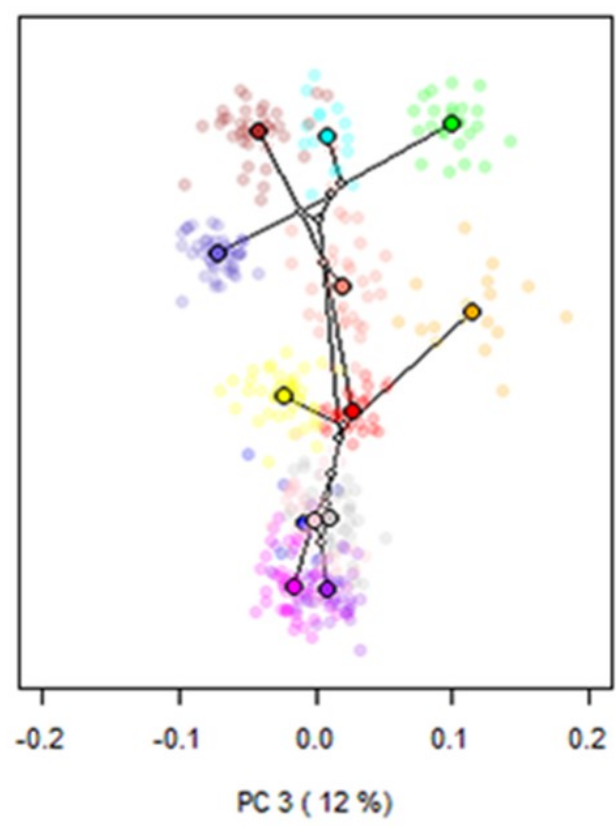

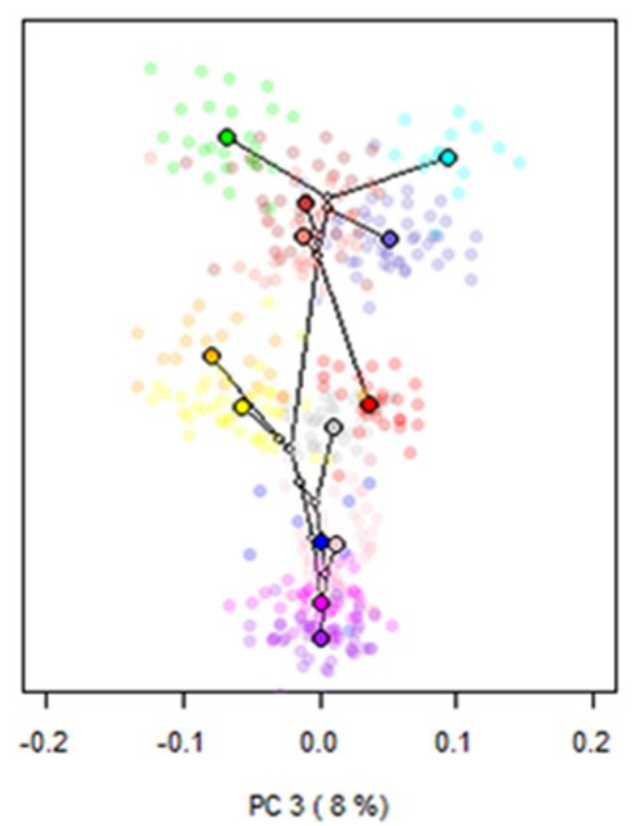

Species

\begin{tabular}{|c|c|c|c|}
\hline $\begin{array}{l}\text { L. bantingensis } \\
\text { L. belanaki } \\
\text { L. careyensis } \\
\text { L. chelatus }\end{array}$ & $\begin{array}{l}O \text { L. fenestrum } \\
O \text { L. funnelus } \\
O \text { L. grandis } \\
O \text { L. johorensis }\end{array}$ & $\begin{array}{l}\text { O L. kedahensis } \\
\text {. L. kederai } \\
\text { OL. liewi } \\
\text { L. navjotsodhii }\end{array}$ & parvi \\
\hline
\end{tabular}


a)

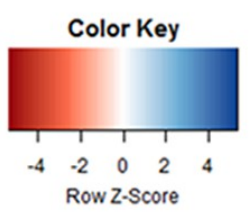

Species

\begin{tabular}{|l|}
\hline OL bantingensis \\
OL belanaki \\
L careyensis \\
OL chelatus \\
OL fenestrum \\
$O$ L funnelus \\
$O$ L grandis \\
$O$ L johorensis \\
$O$ L kedahensis \\
$O$ L kederai \\
$O$ L liewi \\
$O$ L navjotsodhii \\
$O$ L parvicopulatrix
\end{tabular}

503

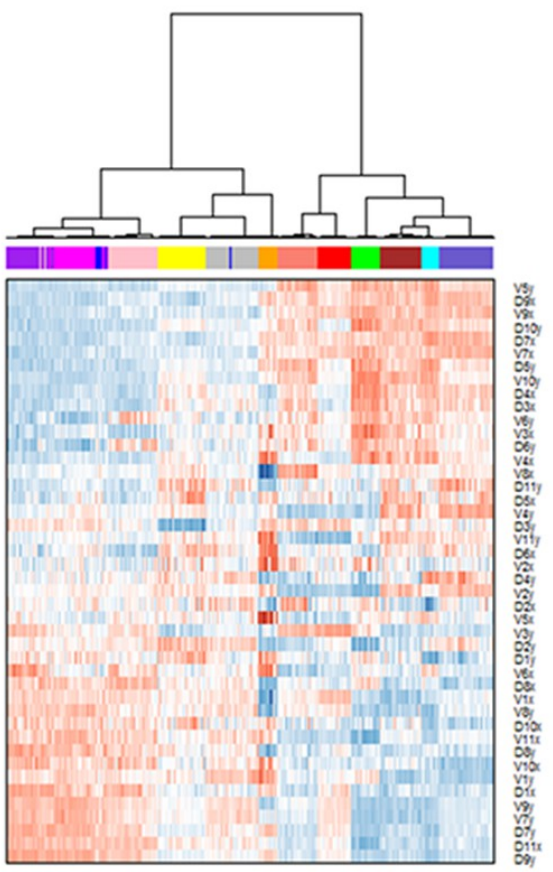

Specimens b)

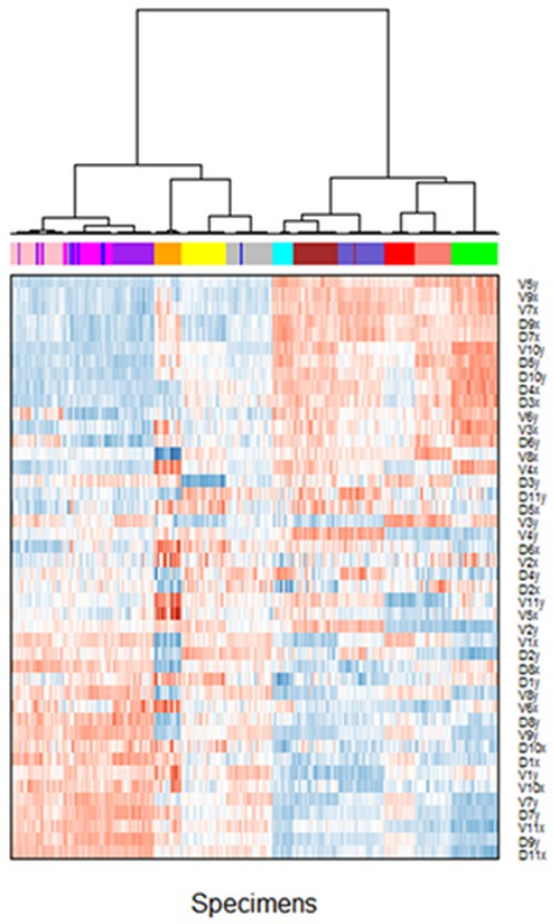

FIGURE 6. improved especially for species with anchors that have very similar shapes such as $L$. 
508 navjotsodhii and L. chelatus (Misclassification error of 2.5\% with filtering (Table S6); $3.8 \%$ with 509 no filtering (Table S7)).

510 For each shape variable, we labelled the samples according to their membership in Clade I

511 or Clade II, and then ranked the shape variables in descending order using the two-sample t-

512 statistic to reveal inverted block structures at the top and bottom of the heat map. The shape

513 variables that make up the top block come from the x-coordinates of LM2, LM4, LM7, LM9, and

$514 \mathrm{y}$-coordinates of LM5, LM6, LM10, their values being relatively positive in species belonging to

515 Clade II compared to those in Clade I. The bottom block consists of shape variables from the x-

516 coordinates of LM1, LM6, LM10, LM11 and y-coordinates of LM1, LM7, LM8, LM9. These

517 values were relatively negative in species belonging to Clade II compared to those in Clade I.

518 Collectively, these variables suggest that the mean shape of the anchor shaft in Clade II was more

519 elongated and scimitar-like (i.e. LM5, LM6, LM10 and LM7, LM8, LM9 are relatively farther

520 from each other) while that in Clade I was more robust and sickle-like (i.e. LM5, LM6, LM10

521 and LM7, LM8, LM9 are relatively closer to each other). 
a)

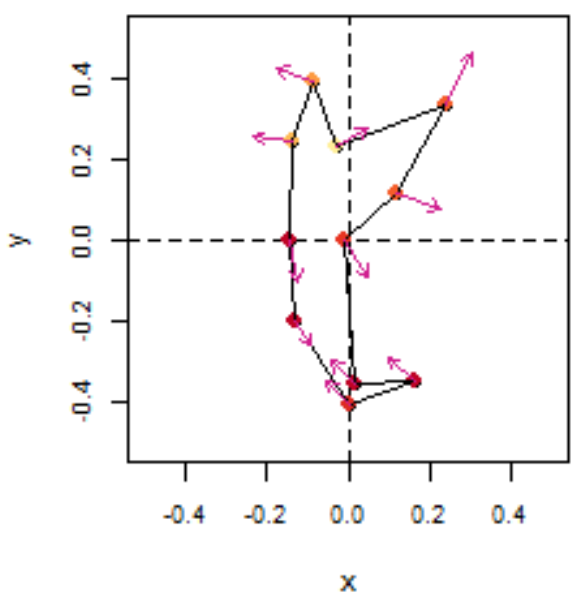

c)

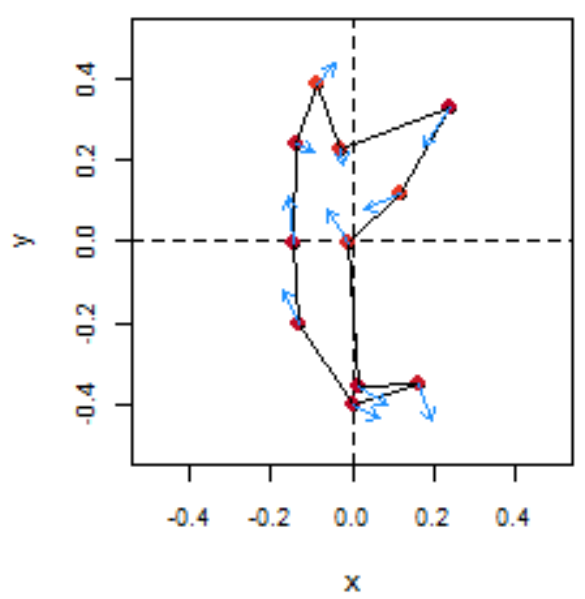

522

523

524

525

526

527

528

529

530

\section{Anchor Shape and Size Evolution}

b)

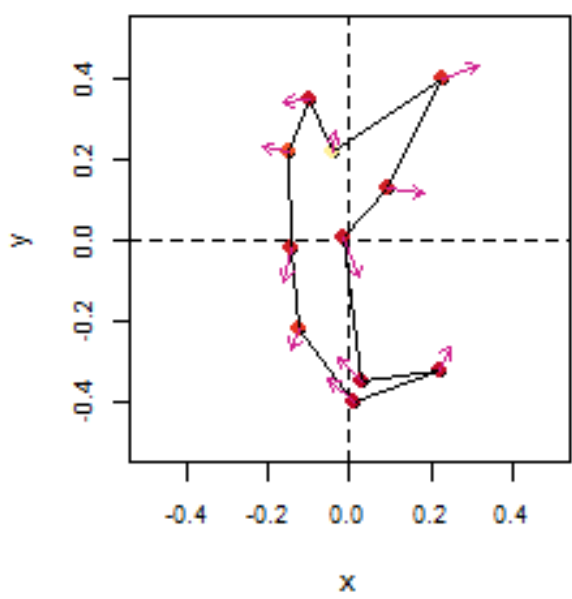

d)

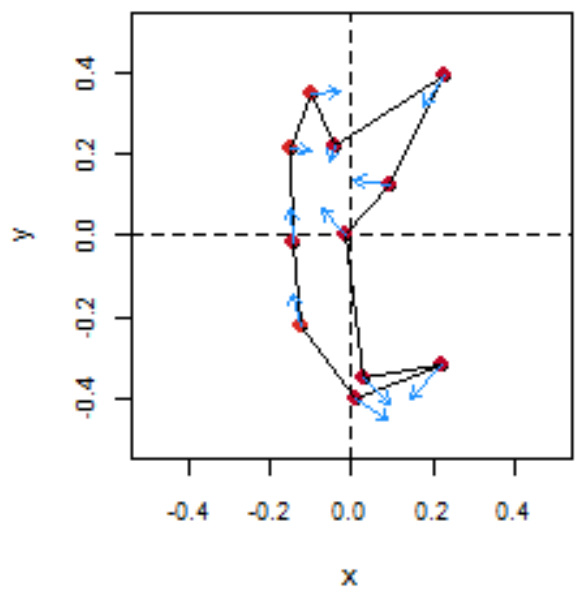

FIGURE 7.
$1.0-$
$0.9-$
$0.8-$
$0.7-$
$0.6-$
$0.5-$

0.4

0.3

0.2

0.1

0.0

The wireframe-lollipop graphs (Fig. 7) show patterns of shape changes in the ventral and

dorsal anchors of both clades that are consistent with those inferred from the cluster heat map and

PCA. The circular plots (Fig. S20 and S21) provide more details at the level of individual

landmarks. The explicit visualization of the direction and magnitude of GPA-landmark coordinate

deviation relative to the ancestral form provides insights into selection of new morphometric

variables suitable as morphological characters. Specifically, for two landmarks, if their mean

directional change is divergent in one clade but convergent in another, then the interlandmark 
531 distance is expected to be of value for discriminating the two clades. To be easy to measure, the 532 landmarks should be of Type I. Thus, the distance from LM1 to LM3 and from LM1 to LM5 533 were found to be good candidates. We found the common practice of using the inner root length 534 (IL) and the outer root length (OL) (distance from LM1 to LM7 and LM3 to LM7, respectively) 535 to be suboptimal since both LM3 and LM7 had almost parallel directional changes (Figs. 7a, 7c, 536 7d), whereas mean directional change in LM1 and LM7 did not show any clear patterns of 537 divergence in one clade and convergence in another (Fig. 7) to be able to show large variation 538 between Clade I and Clade II. Figure 8 (see also Fig. S22 and Fig. S23) shows that it is possible 539 to define cut-offs for the LM1-LM3 $(15 \mu \mathrm{m})$ and LM1-LM5 $(25 \mu \mathrm{m})$ distances that result in 540 discrimination of Clade I from Clade II, but no reasonable cut-offs for IL and OL lead to similar 541 results. 
a)

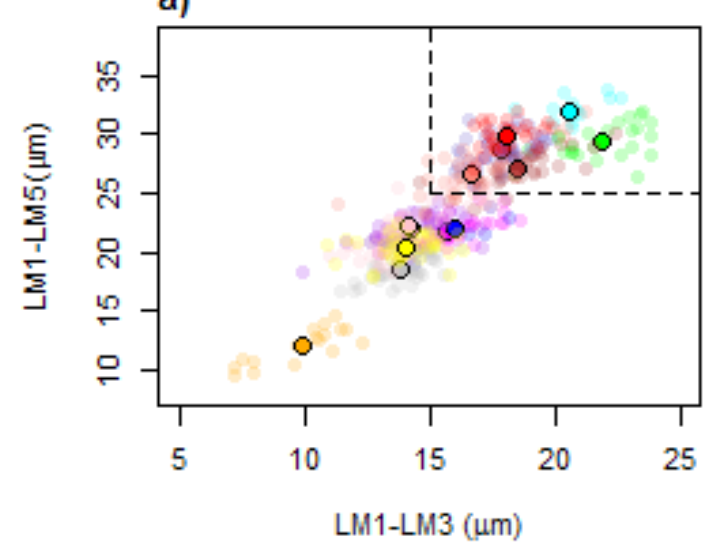

c)

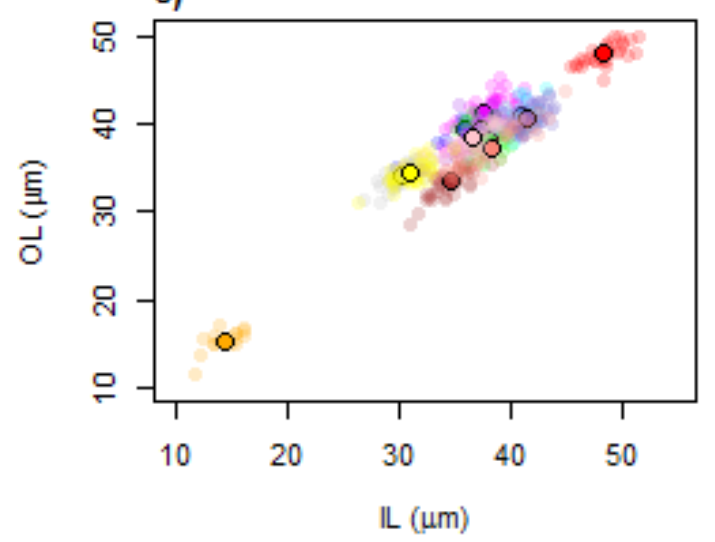

b)

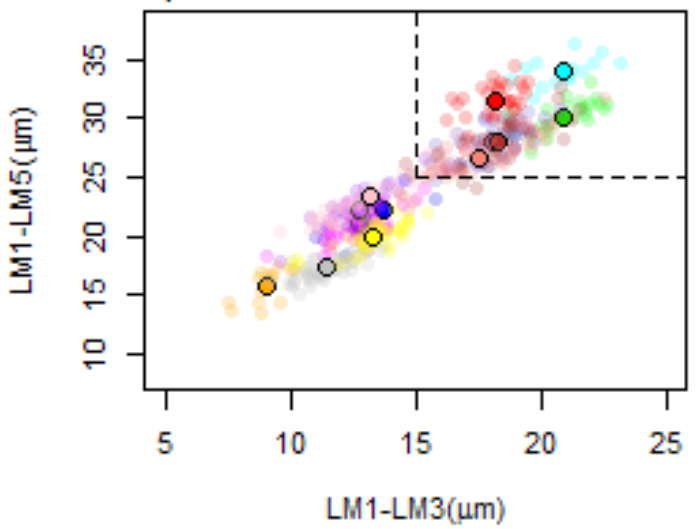

d)

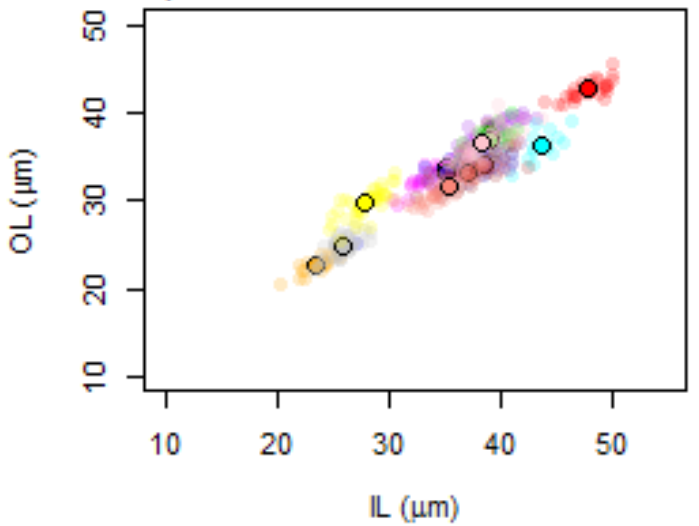

Species

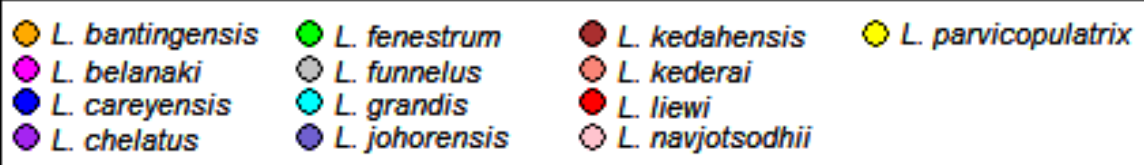

543 The average median body size of species in Clade I was significantly larger compared to

544 Clade II (3.2 times; 95\% confidence interval body size ratio $=[1.5,7])$. However, larger body

545 size was not correlated with larger ventral or dorsal anchor size, after controlling for the effect of

546 phylogeny (Ho-Ané phylogenetic regression p-values $>0.2$ ). Consequently, both of them could

547 be treated as independent covariates. The species in Clade I generally had larger anchors with

548 sickle-shaped shaft (Fig. 9; ventral anchor mean $=170 \mu \mathrm{m}$, standard deviation $(\mathrm{SD})=24 \mu \mathrm{m}$;

549 dorsal anchor mean size $=165 \mu \mathrm{m}, \mathrm{SD}=16 \mu \mathrm{m}$ ), whereas those in Clade II had smaller anchors 
550 (ventral anchor mean size $=140 \mu \mathrm{m}, \mathrm{SD}=35 \mu \mathrm{m}$; dorsal anchor mean size $=133 \mu \mathrm{m}, \mathrm{SD}=24$

$551 \mu \mathrm{m})$ with scimitar-shaped shaft. Size decrease was most striking in L. bantingensis, being 2.7 SD

552 and 1.8 SD below the mean of all species for the ventral and dorsal anchors, respectively.

553 Conversely, size increase was most prominent in L. liewi, with 1.7 SD above the mean of all

554 species for both ventral and dorsal anchors. Nonetheless, the within clade trajectory for some

555 species may sometimes show considerable variation from a clade's average trajectory. For

556 example, L. liewi evolved a more slender shaft for its ventral and dorsal anchors, which is closer

557 to the scimitar shape found in most species in Clade II, even though its anchor size was the

558 largest. In contrast, L. bantingensis evolved sickle-shaped shafts in its ventral and dorsal anchors

559 (common in Clade I species like L. fenestrum and L. grandis) even though it had the smallest

560 anchor size. 


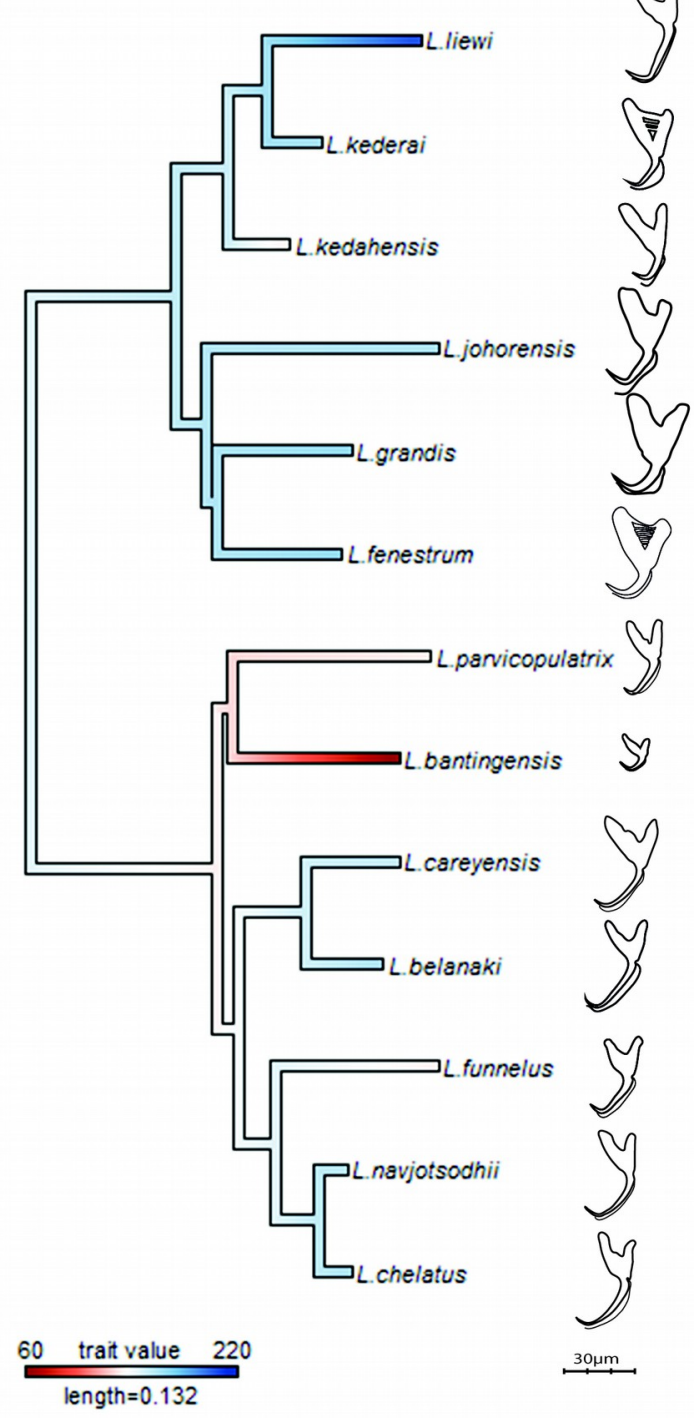

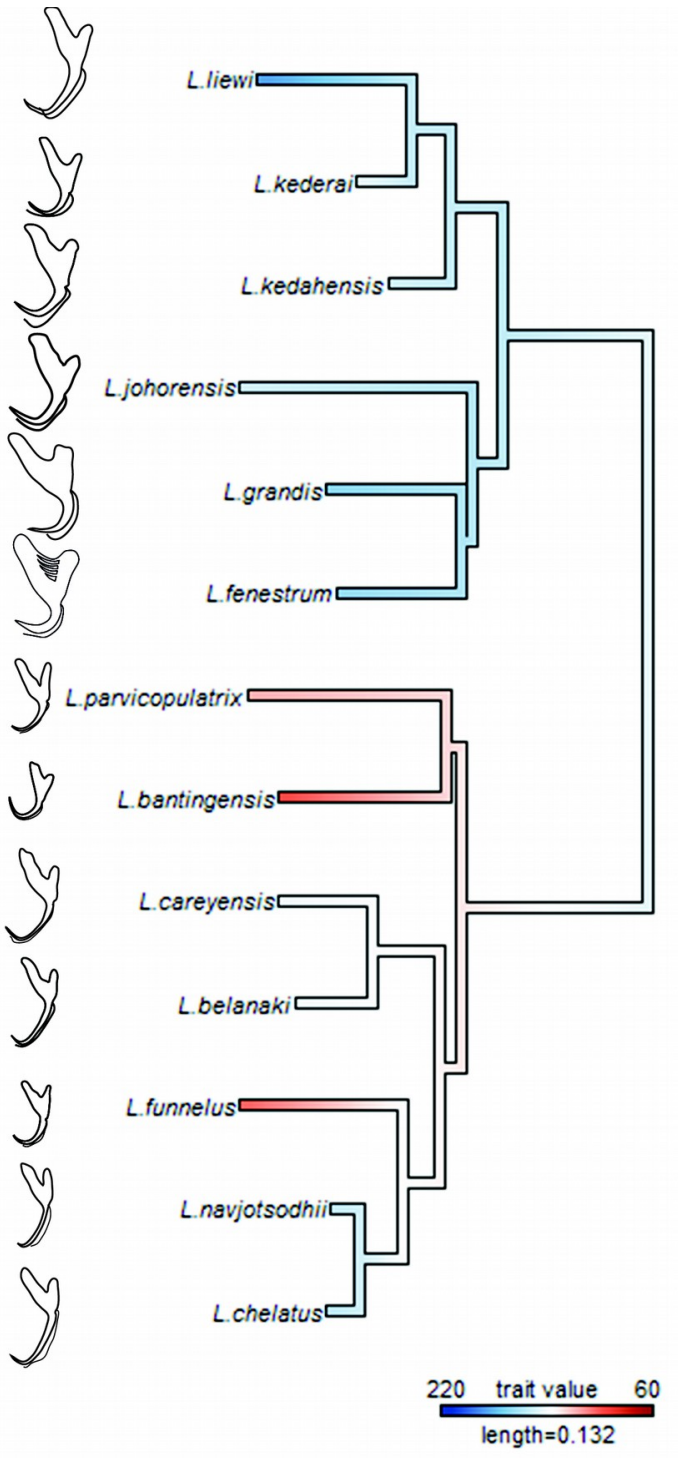

562 Results from the Adams-Collyer phylogenetic regression indicated that the interaction of

563 body size and anchor size was not statistically significant in the dorsal anchor ( $\mathrm{p}$-value $=0.2)$, but 564 anchor size was a significant predictor of anchor shape $(\mathrm{p}$-value $=0.01)$. For the ventral anchor,

565 the interaction of body size and anchor size was a significant predictor of anchor shape (p-value $=$

566 0.02). Since body size and anchor shape were not significantly correlated, we may expect similar 567 anchor shape to be found across a range of body sizes (Fig. S24). 


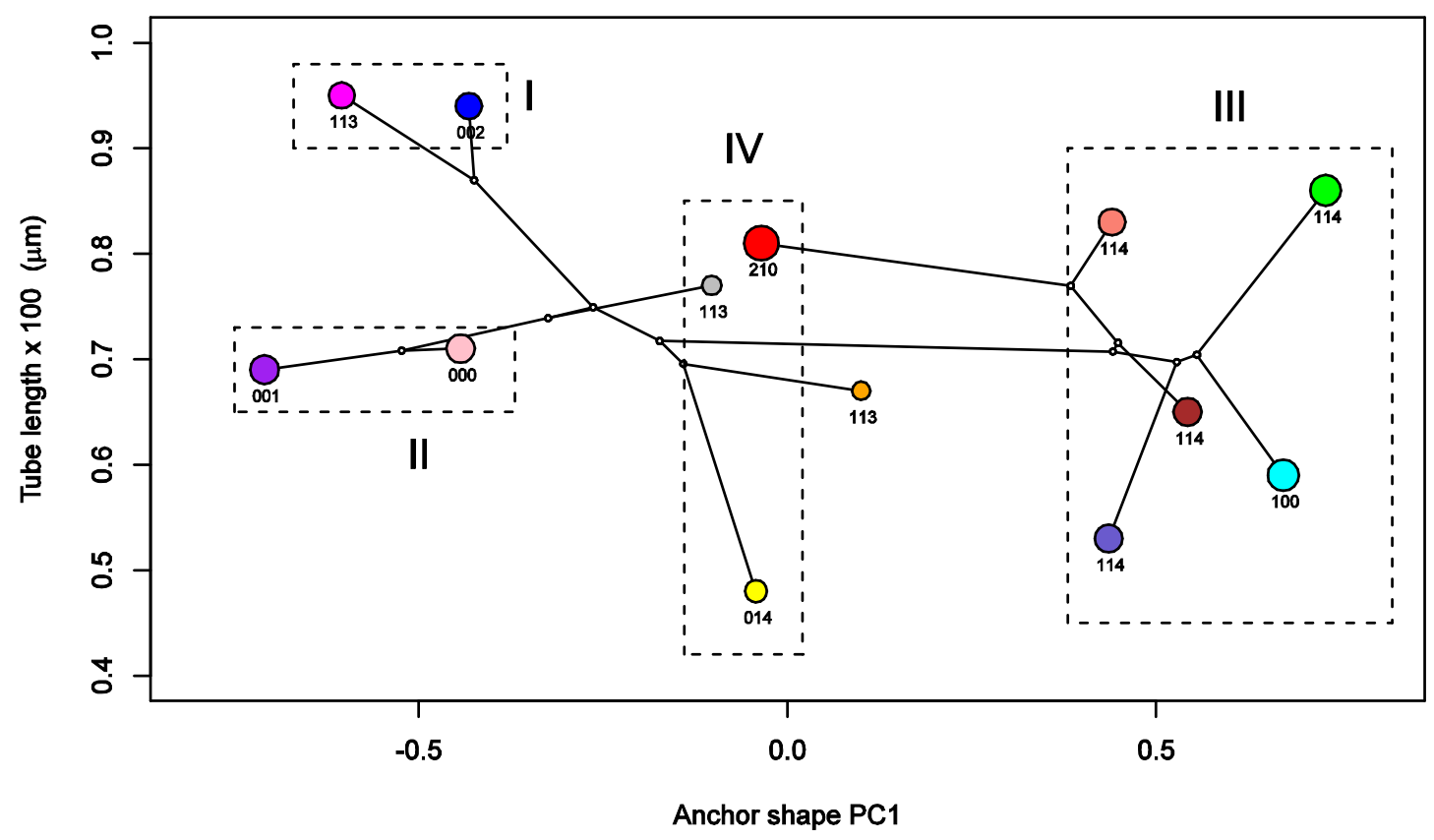

Species

\begin{tabular}{|llll|}
\hline O L. bantingensis & O L. fenestrum & O L. kedahensis & O L. parvicopulatrix \\
O L. belanaki & O L. funnelus & O L. kederai & \\
L. careyensis & O L. grandis & O L. liewi & \\
O L. chelatus & O L. johorensis & O L. navjotsodhii & \\
\hline
\end{tabular}

Patterns of Morphometric and Morphological Variation in the Male Copulatory Organ and

571 Where the male copulatory organ was similar in size among closely-related species with

572 similar anchor shape and size, its morphology varied (I and II in Fig.10). Ligophorus belanaki, L.

573 careyensis, L. navjotsodhii and L. chelatus shared a most recent common ancestor, whose

574 ancestral character states for the three male copulatory organ characters could be inferred as 113

575 on a parsimony criterion. The divergence of L. careyensis from L. belanaki did not involve major 
576 changes in anchor shape, size and size of male copulatory organ, but on the latter's morphology,

577 which acquired three changes to become 002. Similarly, The most recent common ancestor of $L$.

578 navjotsodhii and L. chelatus probably evolved character states 000 or 001 from 113, and

579 divergence of these two species was associated with a change in the third character state, with

580 only relatively minor change in either anchor shape and size or copulatory organ size.

581 In contrast, where the male copulatory organ was similar in morphology among closely-

582 related species with similar anchor shape and size, its size varied (III in Fig. 10). Ligophorus

583 kederai, L. grandis, L. kedahensis and L. johorensis have similar anchor shape and the same

584 character states 114 for the morphology of their male copulatory organ. Consistent with

585 prediction from the Rohde-Hobbs hypothesis, substantial variation in the size of their male

586 copulatory organ was observed. It is possible for size and morphological variation to co-occur in

587 the male copulatory organ, as shown in the divergence of L. grandis and L. fenestrum from their

588 common ancestor. Finally, species with similar anchor shape (IV in Fig. 10) showed large

589 variation in both their male copulatory organ size and morphology.

\section{Morphological Integration}

591 The shapes of both ventral and dorsal anchors were strongly and significantly correlated

592 (evolutionary correlation $=0.84$, Adams-Felice test $p$-value $=0.003$ ). Additionally, there was

593 tight integration between the root and point compartments of the ventral (evolutionary correlation

$594=0.85$, Adams-Felice test $\mathrm{p}$-value $=0.004)$ and dorsal anchors (evolutionary correlation $=0.86$,

595 Adams-Felice test $\mathrm{p}$-value $=0.001)$. Thus, the entire anchor can be considered as a single, fully

596 integrated module. Across the ventral and dorsal anchors, integration of the point compartments

597 was strong (evolutionary correlation $=0.92$, Adams-Felice test $p$-value $=0.0001$ ) but that of the

598 root compartments was weaker (evolutionary correlation $=0.79$, Adams-Felice test $\mathrm{p}$-value $=$ 
599 0.03). Figure S25 provides a graphical summary of the results of the morphological integration 600 analysis.

601 Phylogenetically Informative Morphometric Variables

For the current 13 Ligophorus species, the maximum parsimony tree estimated using the 603 set of morphological characters containing discretized LM1-LM3 and LM1-LM5 distances and 604 anchor shape was better resolved (Fig. 11). Clade I and Clade II were clearly identified, and the 605 partially resolved relationships within each clade were also congruent with those of the molecular 606 phylogeny's. In contrast, using morphological characters of the anchors derived from traditional 607 morphometrics as in Sarabeev and Desdevises (2014) produced a maximum parsimony tree that 608 was mostly reticulate and unable to distinguish Clade I and Clade II.

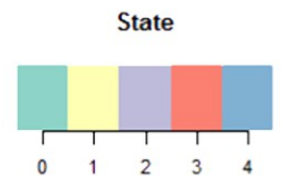

a)

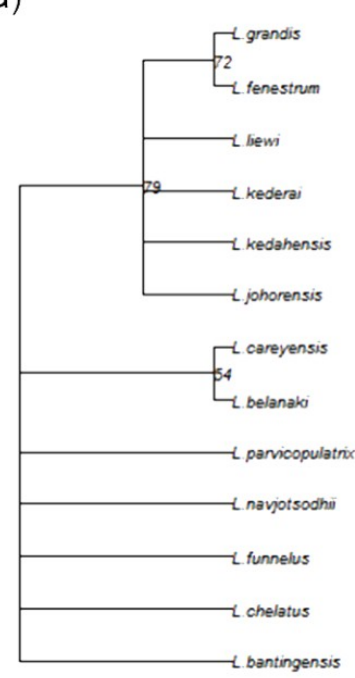

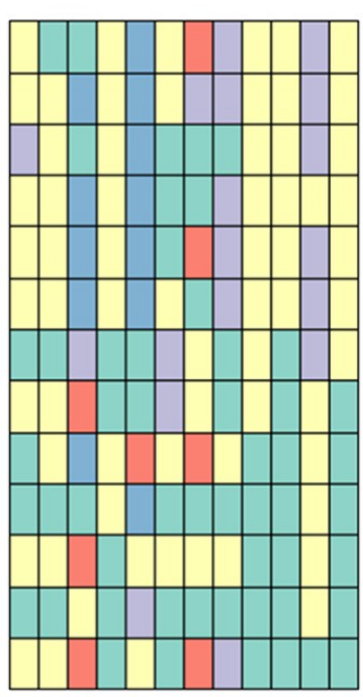

$-a n-\infty, r \infty a=\simeq$

Character b)
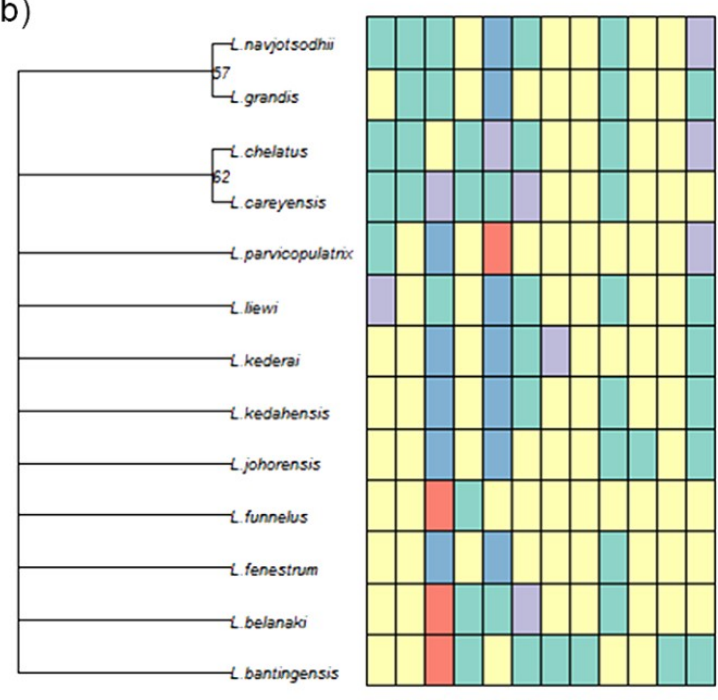

Character 
DISCUSSION

611

612

613

614

615

616

617

\section{Data Quality Control}

We are not aware of any geometric morphometric analyses of anchors in monogeneans that currently implement specimen quality control. Specimen quality introduces an important source of non-biological variation into observed anchor shape variation, the impact of which depends on whether the data would be analyzed at the intra or interspecific level. Thus, while the inclusion of specimens that failed quality control into hierarchical clustering did not

fundamentally change species discrimination outcome in this study, it is important to control for this confounder where intraspecific variation can be expected to impact conclusions of an analysis, for example, when investigating mean directional change in landmarks of anchors (Fig. 7), or testing for association between intraspecific anchor shape variation and evolutionary potential of a species (Rodríguez-González et al., 2015a) . In the current study, we observed up to $50 \%$ loss of specimens (L. fenestrum) due to low quality score. Assuming this value optimistically as an upper bound, then at least 40 specimens per species would have to be obtained in order to anticipate 20 or more specimens passing quality control. An ideal case like this may not always be possible, since sampling trips do not always yield sufficient study material.

used in the quality control procedure has a caveat that should be noted. Poor quality specimens that arise as a consequence of incongruent image and object planes are confounded with specimens that show fluctuating asymmetry. If fluctuating asymmetry (Graham et al., 2010) is common, then a large proportion of specimens would have been discarded using the quality control procedure, but this is not the case in the present study, where about $82 \%(437 / 530)$ of all specimens were retained. Careful observations over multiple well-prepared mounts should help 
634 researchers decide the appropriateness of implementing the quality control step for specimens 635 from a particular monogenean genus.

636 Variation in quality scores can be attributed to several sources, such as the method of slide 637 preparation, the quality of camera lens and software used for capturing images, and the skill and 638 experience of the data gatherer. In this study, a single data gatherer (O.Y.M. Soo) prepared and 639 acquired the landmark data, using the same compound microscope and computer. Because of this, 640 we expect other factors to explain the poor quality scores. Interestingly, we note that species that 641 had larger proportion of specimens failing quality control tend to have body and/or anchor size 642 that were relatively large or small. In the case of L. grandis ( $\log _{10}$ median body size 2.1 SD larger 643 from total mean; dorsal anchor size 1.0 SD larger than total mean), L. fenestrum $\left(\log _{10}\right.$ median 644 body size 1.6 SD larger than total mean; dorsal anchor size 0.9 SD larger than total mean), and $L$. 645 bantingensis ( $\log _{10}$ median body size 0.9 SD smaller than total mean; ventral anchor size 2.7 SD 646 smaller than total mean), we observed about $30 \%, 50 \%$ and $45 \%$ of the specimens failing 647 quality control (Q-score $<10$; Fig. S2), respectively. A possible explanation may be that the 648 robust anchors of L. fenestrum and L. grandis have uneven thickness at the root and point 649 regions, which makes them difficult to evenly flatten on slides. The large body bulk may also 650 further hinder effective flattening. Monogenean anchor thickness is not usually measured but may 651 be indirectly inferred through 3D-modelling (Teo et al., 2010; Teo et al., 2013). Since size and 652 physical inertia are positvely correlated, the small body and anchor size of L. bantingensis make 653 specimen orientation on the slide sensitive to variation in force applied during slide flattening. 
656 in this study. While within species shape variation in both ventral and dorsal anchors was large in

657 some species (L. kedahensis, L. parvicopulatrix), it was limited in others (L. grandis, L. liewi).

658 Interestingly, the generalist L. bantingensis, which has been reported to be found in two small

659 fish hosts (Kritsky et al., 2013): Liza abu (body length range: 12-15.5 cm) and Liza klunzingeri

660 (body length range: $14-18 \mathrm{~cm}$ ), had the largest intraspecific shape variation in its ventral anchor,

661 particularly in its root compartment (PC2). Phenotypic plasticity within species likely promotes

662 divergence by increasing the adaptability to different gill microhabitats (Rohde and Watson,

663 1985; Poisot and Desdevises, 2010; Pfennig et al., 2010; Rodríguez-González et al., 2015a), and

664 is generally considered to be important for generalist species (van Valen, 1965; Kaci-Chaouch et

665 al., 2008; Šimková et al., 2013).

666

667

668

669

670

671

672

673

674 675
Integrative Geometric Morphometric Analysis Supports the Rohde-Hobbs Hypothesis

Evidence for supporting the Rohde-Hobbs hypothesis has traditionally come from integrating spatial distribution data of monogeneans on gill microhabitats (e.g. Rohde, 1977;

Ramasamy et al., 1985; Koskivaara et al., 1992) with morphological data of the monogenean species (e.g. Fig. 6.3 in Šimková and Rohde, 2013). These efforts were very laborious, but crucially established anchor shape-microhabitat association. Benefitting from such insights, our current integrative geometric morphometric analysis was able to reveal patterns consistent with the hypothesis's predictions on how male copulatory organ size and morphology vary with respect to anchor shape (Fig. 10), despite the absence of spatial distribution data for the 13 Ligophorus species across gill microhabitats. 
678 compartments in L. cephali, Rodríguez-González et al. (2015a) reported only modest degree of 679 integration in the same anchor, but stronger compartmental integration between the ventral and 680 dorsal anchors. On the other hand, using interspecific data, we demonstrated a much stronger 681 degree of integration between the root and the point compartments within anchors, and showed relatively weaker integration of the root compartments between ventral and dorsal anchors.

Intuitively, intraspecific compartmental integration within the same anchor is expected to be high, so a possible explanation for the discrepancy may be the lack of a quality control procedure for filtering poor quality slides. Without the latter, it seems difficult to rule out the possibility that the observed intraspecific anchor shape variation in L. cephali may contain non-trivial amount of artifactual noise.

Generally, a certain degree of homoplasy may be expected in the morphology of attachment organs in parasites, on grounds that functional requirements for attaching to the host and adapting to within-host microhabitats would override shape constraints imposed by phylogeny (Morand et al., 2002). Such form of adaptive evolution can cause $\mathrm{K}_{\text {mult }}$ to become less than 1 (Blomberg et al. 2003). In the present Ligophorus phylogeny, the $\mathrm{K}_{\text {mult }}$ value of 0.948 is significantly larger than 0 (p-value 0.0003 ), but slightly biased downwards from the expected value under Brownian motion evolution for anchor shape. The reason seems to be that, although anchor shape is more or less lineage-dependent in the present set of Ligophorus species, three species: L. liewi (Clade I), L. funnelus (Clade II) and L. parvicopulatrix (Clade II), have similar

697 shaft shape (Fig. 9), despite being from different lineages, thus lowering $\mathrm{K}_{\text {mult. }}$ To put the magnitude of $\mathrm{K}_{\text {mult }}$ in perspective, its univariate version - Blomberg's K statistic, has been found

699 to be generally less than 1 for numerous primate traits, exceeding 1 only for brain size (Kamilar 700 and Cooper, 2013). 

and Lindenstrøm, 2002), ecology (Šimková et al., 2006), and behaviour of the fish hosts (Cable et

703 al., 2002; Mendlová and Šimková, 2014 ) all contribute to determine patterns of infection and

704 transmission, host specificity, and subsequent mode of speciation in their monogenean parasites

705 (Littlewood et al., 1997; Cribb et al., 2002; Whittington and Kearn, 2011; Vanhove and Huyse,

706 2015). Therefore, it is not surprising that different monogenean families may show different

707 degrees of morphology-phylogeny covariation. In Lamellodiscus (Family: Diplectanidae), the

708 lack of cospeciation between host-parasite (Desdevises et al., 2002) suggests that adaptation to

709 host after host-switching would lead to convergent evolution in some of the morphologies (but

710 see Machkewskyi et al., 2014). Indeed, Poisot et al. (2011) found morphological features of

711 sclerotized haptor and male copulatory organs to be weakly associated with phylogeny in

712 Lamellodiscus. On the other hand, in Cichlidogyrus (Family: Ancyrocephalidae), sclerite

713 (including anchors) shape was found to contain significant phylogenetic signal (Vignon et al.,

714 2011). However, this conclusion needs to be qualified in light of the recent demonstration of a

715 host-switching event in Cichlidogyrus (Messu Mandeng et al., 2015), which was missed in

716 Vignon et al. (2011) because the host range (at the familial level of the fish hosts) of

717 Cichlidoygrus was not taken into consideration. For Ligophorus, the significant phylogenetic

718 signal found in the anchors does not appear to need similar qualification, since its host range is

719 currently known to be restricted to mullets. In an examination of over 1000 fish from 10 fish

720 families in the Black Sea, Öztürk and Özer (2014) observed that Ligophorus was restricted to

721 mullets (Mugil cephalus and Liza aurata), with almost 100\% prevalence in the hosts.

722 Nevertheless, since it has been reported that a combination of host-switching (within fish host

723 family) and intra-host speciation events probably generated the present diversity of Ligophorus

724 species in the Mediterranean basin (Blasco-Costa et al., 2012), a more definitive conclusion 
725 regarding phylogenetic signal in the anchors requires additional tests using samples of

726 Ligophorus species from that region.

727 The anchor morphology-phylogeny correlation shown for Ligophorus in the present study

728 suggests new morphological variables from the anchors that are potentially useful in

729 morphometric analysis and also morphological phylogenetics. In particular, we suggest more

730 active exploration of the newly proposed morphometric variables: the distance between the inner

731 root point and the outer root point (LM1 - LM3), and the distance between the inner root point

732 and the dent point (LM1 - LM5), as alternatives to existing usage of the inner root length (IL)

733 and the outer root length (OL) in traditional morphometric analysis. The discretization of the two

734 proposed morphometric variables: anchor shaft shape and anchor shaft shape, can also be further

735 considered in future morphological phylogenetic analysis.

736

737

738

739

740

741

742

743

744

745

746

747

\section{Anchor Shape and Size Correlation with Host and Ecological Factors}

In the present study, the modest levels of anchor shape-size covariation revealed through the Adams-Collyer phylogenetic regression analysis for the 13 Ligophorus species suggest that, apart from the effect of shared ancestry, anchor shape-size covariation is likely non-trivially constrained by additional factors, one of which could be their biomechanical compatibility. Another factor is host size and ecology. On average, the larger Moolgarda buchanani (body length range $35-48 \mathrm{~cm}$ ) harbored larger Ligophorus species, whereas the smaller Liza subviridis (body length range $25-30 \mathrm{~cm}$ ) harbored smaller Ligophorus species.

The current analysis showed that L. bantingensis evolved anchors of a smaller size but retained the sickle-shape shaft common in Clade I, which is associated with larger anchor size. The observed small anchor size is consistent with the hypothesis that small or medium-sized attachment organs are generally associated with a generalist lifestyle, as these sizes expand the 
748 host range of monogeneans to small or medium-sized hosts, which are generally more diversified

749 than larger hosts (Morand et al., 2002).

750 In the present study, open seas (Langkawi Island) yielded only M. buchanani samples

751 whereas sheltered marine environment yielded both M. buchanani and Liza subviridis samples.

752 This suggests that perhaps the larger and more robust anchors in Ligophorus species infecting

753 M.buchanani may be a consequence of the hosts' adaptation to rough open seas, where strong

754 water currents are expected. In such conditions, burst swimming is an important locomotory

755 strategy for the fish host, not only to negotiate strong currents, but also to chase swift preys and

756 avoid strong predators (Koehn and Crook, 2013). Indeed, for skin-parasitic fish monogeneans,

757 their attachment appendages must be able to withstand dislodgement by strong water currents

758 pushing against the fish surface as the fish host performs burst swimming (Kearn, 2014).

759 Concurrently, it seems plausible that burst swimming may also, for a short time, cause strong

760 water currents to flow through the gill chamber, thus imposing a selective pressure for more

761 robust anchors in monogeneans that live on the gills.

762 The U-shaped root groove in some species infecting M. buchanani (e.g. L. liewi, L.

763 kederai and L. fenestrum), which could result from the accretion of sclerotic material in the space

764 between the inner and the outer root (Klaus Rohde, pers. comm., 2014), may have substantially

765 expanded the root base, providing space for connection with more muscle tissues. This would

766 likely have resulted in anchors with stronger contraction strength, necessitating the evolution of

767 the shorter but more robust sickle-shaped shaft, hence the finding of tight morphological

768 integration between the root and point compartments. species (Vignon et al., 2011), which infect the cichlids in Africa. Ecologically, it seems unlikely

771 that such robust shapes would have evolved in freshwater environments, where cichlids are

772 mostly found. If the ancestors of cichlids and their monogenean parasites were marine in origin, 
773 or host switching occurred with contact between salt-tolerant cichlids and the marine ancestor of

774 the monogenean parasite, the observed robust shapes would then be inherited, with their shapes

775 constrained by phylogeny. While some studies (Murray, 2001) suggested that the ancestors of

776 cichlids were likely marine, others were sceptical (Chakrabarty, 2004; Sparks and Smith, 2005).

777 Joint consideration of the morphology and phylogeny of monogenean fauna of cichlids and other

778 marine fishes is probably required (Pariselle et al., 2011) to assess the competing claims. For

779 example, a recent molecular phylogenetic analysis using 28S rRNA sequence (Tan, 2013; Fig.

780 S26) indicated that Ligophorus (marine) and Cichlidogyrus (mostly freshwater) species shared

781 the most recent common ancestor.

782 In three species (L. grandis, L. funnelus and L. liewi), fenestration in the anchor base

783 seems to be an invariant character state, as all examined specimens ( $n=22,50,32$, respectively)

784 showed consistent presence of fenestration. Presently, the ecological significance of fenestrations

785 in anchors is unclear. Some progress may be possible with biomechanical studies (e.g. Wong and

786 Gorb, 2013) that compare whether fenestrated and non-fenestrated anchors differ significantly in

787 their resistance against turbulence and strong water currents. The present phylogenetic analysis

788 suggests that fenestration is not a synapomorphic character state (Fig. 3), but a clearer picture

789 requires more extensive taxa sampling.

790 Outlook for Geometric Morphometric Analysis of Sclerotized Haptoral Structures in

791 Monogenean Parasites

792 The use of less sophisticated methods could have also answered the biological problems

793 that were studied in the present work. For example, to infer the presence of phylogenetic signal in

794 attachment organs, or to investigate anchor size correlation with parasite and host phylogeny, one

795 could map qualitative shape and size categories of the organ of interest onto the molecular

796 phylogeny of the parasites, as done in Šimková et al. (2006). Validation of the Rohde-Hobbs 
797 hypothesis could be done by correlating parasite abundance data in different gill microhabitats

798 with their attachment organ and male copulatory organ morphology. The lack of a unified

799 quantitative framework, however, forces the researcher to use different data types to address each

800 question. We have shown in the present work that, if geometric morphometric data is used as a

801 starting point, all the preceding questions can be simultaneously answered. In fact, the geometric

802 morphometric approach is the key that unlocks the potential of shape information in the organs of

803 interest for broader research questions, such as species and biogeographical population

804 discrimination (Vignon and Sasal, 2010), morphological integration (e.g. Vignon et al. 2011;

805 Rodríguez-González et al., 2015a), and more recently, canalization and developmental stability

806 (Llopis-Belenguer et al. 2015), all of which cannot be answered satisfactorily using traditional

807 morphometric or qualitative methods.

808 Our present study used large numbers of samples, averaging about 35 per species.

809 Compared to laborious measuring of selected lengths as done in traditional morphometrics, data

810 acquisition is far more efficient with a landmark digitization software such as TPSDIG2, which

811 simultaneously captures shape and size variation information. This improved efficiency is

812 important - by greatly reducing the tedium associated with measuring many lengths per

813 specimen, there is more incentive to sample more extensively.

814 Although the geometric morphometric approach has been strongly advocated by Vignon

815 and Sasal (2010) as an effective means to pursue systematics research in monogeneans, the

816 scientific community still lack integrative tools that would make it easy to share data and adopt a

817 common analysis pipeline to ease comparison of old and new results. Here, the monogeneaGM

$818 \mathrm{R}$ package that we have developed enables substantial number of shape and size variables from

819 large number of samples to be analyzed efficiently to answer multiple questions, ranging from

820 systematic value of the sclerotized haptoral structures to understanding patterns of phenotypic 
821 and phylogenetic correlation. We hope the development of monogenea GM will contribute to

822 reducing the bottleneck for large scale data analysis in the study of monogeneans. Indeed, as

823 there has been a surge in alpha taxonomy and systematic biology studies of Ligophorus in recent

824 years (Abdallah et al., 2009; Siquier and Ostrowski de Núñez, 2009; Blasco-Costa et al., 2012;

825 Dmitrieva et al., 2012, 2013; Soo and Lim, 2012, 2015; El Hafidi et al., 2013; Kritsky et al.,

826 2013; Sarabeev and Desdevises, 2014; Marchiori et al., 2015; Rodríguez-González et al., 2015b;

827 Soo et al., 2015), the analysis can only get more interesting as data from other species from other

828 hosts in other geographical regions are added. We expect the data analysis tools in

829 monogeneaGM to continue to evolve to handle complexities of data analysis when this happens.

830 The use of two-dimensional landmark data implies that the analysis of anchor size and

831 shape evolution is necessarily approximate, since some of the potential biological variation in

832 anchor morphometry may only be adequately captured in three dimensions (Galli et al., 2007).

833 Nevertheless, given the wealth of corroborative inference regarding anchor shape and size

834 evolution that have been obtained in the current study, it appears that no general loss of

835 interpretability arises from usage of two-dimensional data for geometric morphometric analysis

836 in Ligophorus.

837 Future Prospects

838 Adequate taxa coverage remains an important factor for accurate phylogenetic inference

839 (Sanderson et al., 2010). The three markers used in this study are the most common ones reported

840 for other known Ligophorus species in the GenBank database, hence their continued use supports

841 efforts at expanding taxa sampling of molecular sequences. Indeed, a research program in

842 Ligophorus systematics that expands taxa coverage of anchor geometric morphometric and

843 sequence data opens up the possibility of using parasite phylogeny and anchor morphometry to

844 test hypotheses of host genealogy and ecology (Nieberding and Olivieri, 2007) in grey mullets 
845 (Teleostei: Mugilidae), a speciose fish family that is economically important (Durand et al.,

846 2012). Moreover, analysis of patterns of congruence between the phylogenies of the fish host

847 species and their Ligophorus parasites can provide insights into prevalence of host switching

848 (Zietara and Lumme, 2002; Vanhove and Huyse, 2015) and thence the relative importance of

849 allopatric and sympatric speciation (Huyse et al., 2003) in shaping the diversity of this genus. It is

850 also possible to expand this analysis in a biogeographic context by sampling different

851 geographical populations of a host species, since some Ligophorus species have been reported to

852 be useful biological markers of geographical fish host populations (El Hafidi et al., 2013). Lastly,

853 we suggest that the present methods of generating and treating geometric morphometric data

854 from the anchors, and possibly other sclerotized hard parts, can be extended to other monogenean

855 families with little difficulty.

856 CONCLUSIONS

857 The presence of significant phylogenetic signal in the anchors makes the quantitative

858 analysis of their shape and size variables useful in answering species discrimination and

859 evolutionary problems in Ligophorus specifically, and in other monogenean genera more

860 generally. In this study, we inferred two major host-specific clades from DNA sequence data,

861 which corroborated well with clades inferred from geometric morphometric data of anchors. We

862 further extracted size information through the first principal component of size variables based on

863 all pairwise Euclidean distances between landmarks, and showed that the Ligophorus species

864 infecting Moolgarda buchanani generally evolved larger anchors compared to those infecting

865 Liza subviridis. Anchor shape was correlated with anchor size after controlling for the effect of

866 phylogeny. Subsequently, through the analysis of directional change, we discovered two new

867 morphological characters based on the length between the inner root length and the outer root

868 length, and the length between the inner root point and the dent point, which proved more 
869 phylogenetically informative than existing characters based on the inner length and the outer

870 length. The Rohde-Hobbs hypothesis was validated in the 13 species of Ligophorus considered,

871 suggesting the exploitation of different microhabitats and subsequent evolution of reproductive

872 barriers in the form of highly variable male copulatory organ size and morphology after intrahost

873 speciation. Finally, we demonstrated evidence for significant interspecific morphological

874 integration of the root and point compartments within the anchors, as well as integration of the

875 same compartment between the ventral and dorsal anchors.

\section{SOFTWARE AVAILABILITY}

877 The monogeneaGM package is available for download at https://cran.r-

878 project.org/web/packages/monogeneaGM/. Analyses in this study can be replicated using the R 879 scripts deposited in Supplemental Material C1.

\section{ACKNOWLEDGMENTS}

This work is dedicated to the memory of Lee Hong Susan Lim (1952-2014; see Gibson and $\mathrm{Ng}, 2014$ ), who conceived the present project. Susan Lim contributed actively to global monogenean systematics for decades, and was instrumental at developing and transmitting this art in Malaysia. Klaus Rohde read the early version of the manuscript and provided helpful feedback. We thank Thian Liang Cheow for contributing R codes for data processing. Maarten Vanhove helpfully pointed out some very recent studies that were useful for the Discussion section. Finally, we are grateful to Jean-Lou Justine (Academic Editor), Timothée Poisot, and an anonymous reviewer for their constructive comments which stimulated important improvements to the present work. 
891 Abdallah V.D., de Azevedo R.K., Luque J.L. 2009. Four new species of Ligophorus (Monogenea:

892 Dactylogyridae) parasitic on Mugil liza (Actinopterygii: Mugilidae) from Guandu River,

893 southeastern Brazil. J. Parasitol. 95: 855-864.

894 Adams D.C. 2014a. A generalized K statistic for estimating phylogenetic signal from shape and 895 other high-dimensional data. Syst. Biol. 63: 685-697.

896 Adams D.C. 2014b. A method for assessing phylogenetic least squares models for shape and 897 other high-dimensional multivariate data. Evolution 68: 2675-2688.

898 Adams D.C., Felice R.N. 2014. Assessing trait covariation and morphological integration on 899 phylogenies using evolutionary covariance matrices. PLoS ONE 9(4): e94335.

900 Adams D.C., Otarola-Castillo E. 2013. geomorph: an R package for the collection and analysis 901 of geometric morphometric shape data. Methods Ecol. Evol. 4: 393-399.

902 Adams D.C., Rohlf F.J., Slice D.E. 2004. Geometric morphometrics: ten years of progress 903 following the "revolution". Ital. J. Zool. 71: 5-16.

904 Adams D.C., Rohlf F.J., Slice D.E. 2013. A field comes of age: geometric morphometrics in the 905 21st century. Hystrix 24: 7-14.

906 Adler D., Murdoch D., Nenadic O., Urbanek S., Chen M., Gebhardt A., Bolker B., Csardi G.,

907 Strzelecki A., Senger A. et al.2014. rgl: 3D visualization device system (OpenGL). R package 908 version 0.95.1201. Available at http://CRAN.R-project.org/package=rgl.

909 Agostinelli C., Lund U. 2013. R package 'circular': Circular Statistics (version 0.4-7). Available 910 at:https://r-forge.r-project.org/projects/circular. 
911 Arnqvist G., Mårtensson T. 1998. Measurement error in geometric morphometrics: empirical

912 strategies to assess and reduce its impact on measures of shape. Acta Zool. Acad. Sci. H. 44: 7391396.

914 Bakke T.A., Harris P.D., Cable J. 2002. Host specificity dynamics: observations on gyrodactylid 915 monogeneans. Int. J. Parasitol. 32: 281-308.

916 Barão K.R., Gonçalves G.L., Mielke O.H.H., Kronforst M.R., Moreira G.R.P. 2014. Species

917 boundaries in Philaethria butterflies: an integrative taxonomic analysis based on genitalia 918 ultrastructure, wing geometric morphometrics, DNA sequences, and amplified fragment length 919 polymorphisms. Zool. J. Linn. Soc. 170: 690-709.

920 Batschelet E. 1981. Circular Statistics in Biology. London: Academic Press.

921 Blair D. 2006. Ribosomal DNA Variation in Parasitic Flatworms. In: Maule A.G., Marks N.J., 922 editors. Parasitic Flatworms: Molecular Biology, Biochemistry, Immunology and Physiology. 923 Wallingford: CAB International. p. 96-123.

924 Blasco-Costa I., Miguez-Lozano R., Balbuena J.A. 2012. Molecular phylogeny of species of 925 Ligophorus (Monogenea: Dactylogyridae) and their affinities within the Dactylogyridae.

926 Parasitol. Int. 61: 619-627.

927 Blomberg S.P., Garland T., Ives A.R. 2003. Testing for phylogenetic signal in comparative data: 928 behavioral traits are more labile. Evolution 57: 717-745.

929 Boeger W.A., Kritsky D.C. 1997. Coevolution of the Monogenoidea (Platyhelminthes) based on a 930 revised hypothesis of parasite phylogeny. Int. J. Parasitol. 27: 1495-1511. 
931 Boeger W.A., Kritsky D.C. 2001. Phylogenetic relationships of the Monogenoidea. In:

932 Littlewood D.T.J., Bray R.A. editors. Interrelationships of the Platyhelminthes. London: Taylor \& 933 Francis. p. 92-102.

934 Buchmann K., Lindenstrøm T. 2002. Interactions between monogenean parasites and their fish 935 hosts. Int. J. Parasitol. 32: 309-319.

936 Cable J., Scott E.C.G., Tinsley R.C., Harris P.D. 2002. Behavior favoring transmission in the 937 viviparous monogenean Gyrodactylus turnbulli. J. Parasitol. 88: 183-184.

938 Chakarabarty P. 2004. Cichlid biogeography: comment and review. Fish Fish. 5: 97-119.

939 Collyer M.L., Sekora D.J., Adams D.C. 2015. A method for analysis of phenotypic change for 940 phenotypes described by high-dimensional data. Heredity 115: 357-365.

941 Conesa M.A., Mus M., Rosselló J.A. 2012. Leaf shape variation and taxonomic boundaries in 942 two sympatric rupicolous species of Helichrysum (Asteraceae: Gnaphalidae), assessed by linear 943 measurements and geometric morphometry. Biol. J. Linn. Soc. 106: 498-513.

944 Cribb T.H., Chisholm L.A., Bray R.A. 2002. Diversity in the Monogenea and Digenea: does 945 lifestyle matter? Int. J. Parasitol. 32: 321-328.

946 Cruz R.A.L., Pante M.J.R., Rohlf F.J. 2012. Geometric morphometric analysis of shape variation 947 in Conus (Gastropoda: Conidae). Zool. J. Linn. Soc. 165: 296-310.

948 De Meeus T., Michalakis Y., Renaud F. 1998. Santa Rosalia revisited: or why are there so many 949 kinds of parasites in 'The Gardern of Earthly Delights'? Parasitol. Today 14: 10-13. 
950 Desdevises Y., Morand S., Jousson O., Legendre P. 2002. Coevolution between Lamellodiscus

951 (Monogenea; Diplectanidae) and Sparidae (Teleostei): the study of a complex host-parasite 952 system. Evolution 56: 2459-2471.

953 Dmitrieva E.V., Gerasev P.I., Gibson D.I., Pronkina N.V., Galli P. 2012. Descriptions of eight new 954 species of Ligophorus Euzet \& Suriano, 1977 (Monogenea: Ancyrocephalidae) from Red Sea 955 mullets. Syst. Parasitol. 81: 203-237.

956 Dmitrieva E.V., Gerasev P.I., Gibson D.I. 2013. Ligophorus abditus n. sp. (Monogenea:

957 Ancyrocephalidae) and other species of Ligophorus Euzet \& Suriano, 1977 infecting the flathead 958 grey mullet Mugil cephalus L. in the Sea of Japan and the Yellow Sea. Syst. Parasitol. 85: 117959130.

Durand J.D., Shen K.N., Chen W.J., Jamandre B.W., Blel H., Diop K., Nirchio M., Garcia de

961 Leon F.H., Whitfield A.K., Chang C.W., Borsa P. 2012. Systematics of the grey mullets 962 (Teleostei: Mugiliformes: Mugilidae): molecular phylogenetic evidence challenges two centuries 963 of morphology-based taxonomy. Mol. Phylogenet. Evol. 64: 73-92.

964 El Hafidi F., Rkhami O.B., de Buron I., Durand J-D., Pariselle A. 2013. Ligophorus species 965 (Monogenea: Ancyrocephalidae) from Mugil cephalus (Teleostei: Mugilidae) off Morocco with 966 the description of a new species and remarks about the use of Ligophorus spp. as biological 967 markers of host populations. Folia Parasitol. 60: 433-440. Mol. Evol. 17: 368-376. 
971 Galli P., Strona G., Villa A.M., Benzoni F., Stefani F., Doglia S.M., Kritsky D.C. 2006. Two-

972 dimensional versus three-dimensional morphometry of monogenoidean sclerites. Int. J. Parasitol.

$973 \quad 37: 449-456$.

974 Gibson D.I., Ng P.T.K. 2014. Lim Lee Hong, Susan (1952-2014) - monogenean systematist and

975 Commisioner 2006-2014. Bull. Zool. Nomen. 71: 217-220.

976 Gilmore S.R., Cone D.K., Lowe G., King S.K., Jones S.R.M., Abbott C.L. 2012. Molecular

977 phylogeny of Gyrodactylus (Monogenea) parasitizing fishes in fresh water, estuarine, and marine

978 habitats in Canada. Canadian J. Zool. 90: 776-786.

979 Gower J.C. 1975. Generalized procrustes analysis. Psychometrika 40: 33-51.

980 Graham J.H., Raz S., Hel-Or H., Nevo E. 2010. Fluctuating asymmetry: methods, theory and 981 applications. Symmetry 2: 466-540.

982 Grégoir A.F., Hablützel P.I., Vanhove M.P.M., Pariselle A., Bamps J., Volckaert F.A.M., 983 Raeymaekers J.A.M. 2015. A link between host dispersal and parasite diversity in two sympatric 984 cichlids of Lake Tangayika. Freshwater Biol. 60: 323-335.

985 Hahn C., Weiss S.J., Stojanovski S., Bachmann L. (2015). Co-speciation of the ectoparasite 986 Gyrodactylus teuchis (Monogenea, Platyhelminthes) and its salmonid hosts. PLoS ONE 10: $987 \mathrm{e} 0127340$.

988 Hahn C., Bakke T.A., Bachmann L., Weiss S., Harris P.D. 2011. Morphometric and molecular 989 characterization of Gyrodactylus teuchis Lautraite, Blanc, Thiery, Daniel \& Vigneulle, 1999

990 (Monogenea: Gyrodactylidae) from an Austrian brown trout population. Parasitol. Int. 60: 480991487. 
992 Hayward C. 2005. Monogenea Polyopisthocotylea (ectoparasitic flukes). In: Rohde K., editor.

993 Marine Parasitology. Australia: CSIRO Publishing. p. 55-63.

994 Ho L.S.T., Ané C. 2014. A linear-time algorithm for Gaussian and non-Gaussian trait evolution 995 models. Syst. Biol. 63: 397-408.

996 Huyse T., Audenaert V., Volckaert F.A.M. 2003. Speciation and host-parasite relationships in the 997 parasite genus Gyrodactylus (Monogenea, Platyhelminthes) infecting gobies of the genus 998 Pomatoschistus (Gobiidae, Teleostei). Int. J. Parasitol. 33: 1679-1689.

999 Huyse T., Volckaert F.A. 2005. Comparing host and parasite phylogenies: Gyrodactylus 1000 flatworms jumping from goby to goby. Syst. Biol. 54: 710-718.

1001 Jarkovský J., Morand S., Šimková A., Gelnar M. 2004. Reproductive barriers between congeneric 1002 monogenean parasites (Dactylogyrus: Monogenea): attachment apparatus morphology or 1003 copulatory organ incompatibility? Parasitol. Res. 92: 95-105.

1004 Jolliffe I.T. 2002. Principal Component Analysis. 2nd ed. New York: Springer-Verlag.

1005 Kaci-Chaouch T., Verneau O., Desdevises Y. 2008. Host specificity is linked to intraspecific 1006 variability in the genus Lamellodiscus (Monogenea). Parasitol. 135: 607-616.

1007 Kahle D., Wickham H. 2013. ggmap: spatial visualization with ggplot2. The R Journal 5(1): 1441008161.

1009 Kamilar J.M., Cooper N. 2013. Phylogenetic signal in primate behaviour, ecology and life 1010 history. Phil. Trans. R. Soc. B 368: 20120341. 
1011 Katoh K., Standley D.M. 2013. MAFFT multiple sequence alignment software version 7:

1012 Improvements in performance and usability. Mol. Biol. Evol. 30: 772-780.

1013 Kearn G.C. 2014. Some aspects of the biology of monogenean (Platyhelminth) parasites of 1014 marine and freshwater fishes. Oceanography 2: 117. doi: 10.4172/2332-2632.1000117

1015 Khang T.F. 2015. monogeneaGM: geometric morphometric analysis of monogenean anchors. R 1016 package version 1.0. Available at: http://CRAN.R-project.org/package=monogeneaGM.

1017 Klingenberg C.P. 2008. Morphological integration and developmental modularity. Annu. Rev. 1018 Ecol. Evol. Syst. 39: 115-132.

1019 Klingenberg C.P. 2013. Visualizations in geometric morphometrics: how to read and how to make 1020 graphs showing shape changes. Hystrix 24:15-24.

1021 Klingenberg C.P., Ekau W. 1996. A combined morphometric and phylogenetic analysis of an 1022 ecomorphological trend: pelagization in Antarctic fishes (Perciformes: Nototheniidae). Biol. J. 1023 Linn. Soc. 59: 143-177.

1024 Koehn J.D., Crook D.A. 2013. Movement and migration. In: Humphries P., Walker K., editors. 1025 Ecology of Australian Freshwater Fishes. Australia: CSIRO Publishing. p. 105-130.

1026 Koskivaara M., Valtonen E.T., Vuori K-M. 1992. Microhabitat distribution and coexistence of 1027 Dactylogyrus species (Monogenea) on the gills of roach. Parasitology 104: 273-281.

1028 Kritsky D.C., Khamees N.R., Ali A.H. 2013. Ligophorus spp. (Monogenoidea: Dactylogyridae) 1029 parasitizing mullets (Teleostei: Mugiliformes: Mugilidae) occurring in the fresh and brackish 1030 waters of the Shatt Al-Arab River and Estuary in southern Iraq, with the description of 1031 Ligophorus sagmarius sp. n. from the greenback mullet Chelon subviridis (Valenciennes). 1032 Parasitol. Res. 112: 4029-4041. 
1033 Lambert A., Maillard C. 1975. Repartition branchiale de deux monogenes: Diplectanum aequans

1034 (Wagener, 1857) Diesing, 1858 et D. laubieri Lambert A., Maillard, C., 1974 (Monogenea:

1035 Monopisthocotylea) parasites simultanes de Dicentrarchus labrax (Teleosteen). Ann. Parasitol.

1036 Hum. Comp. 50: 691-699.

1037 Lim L.H.S. 1991. Three new species of Bychowskyella Achmerow, 1952 (Monogenea) from

1038 Peninsular Malaysia. Syst. Parasitol. 19: 33-41.

1039 Lim L.H.S., Gibson D.I. 2009. A new monogenean genus from an ephippid fish off Peninsular 1040 Malaysia. Syst. Parasitol. 73: 13-25.

1041 Littlewood D.T.J. 2008. Platyhelminth systematics and the emergence of new characters. Parasite 1042 15: 333-341.

1043 Littlewood D.T.J., Olson P.D. 2001. Small subunit rDNA and the Platyhelminthes: Signal, noise, 1044 conflict and compromise. In: Littlewood D.T.J., Bray R.A., editors. Interrelationships of the 1045 Platyhelminthes. New York: Taylor \& Francis. p. 262-278.

1046 Littlewood D.T.J., Rohde K., Clought K.A. 1997. Parasite speciation within or between host 1047 species? Phylogenetic evidence from site-specific polystome monogeneans. Int. J. Parasitol. 27: $1048 \quad 1289-1297$.

1049 Llopis-Belenguer C., Balbuena J.A., Galván-Femenía I., Rodríguez-González A. 2015. 1050 Phenotypic buffering in a monogenean: canalization and developmental stability in shape and 1051 size of the haptoral anchors of Ligophorus cephali (Monogenea: Dactylogyridae). PLoS ONE 10: $1052 \mathrm{e} 0142365$. 
1053 Lockyer A.E., Olson P.D., Littlewood D.T.J. 2003. Utility of complete large and small subunit 1054 rRNA genes in resolving the phylogeny of the Neodermata (Platyhelminthes): implications and a 1055 review of the cercomer theory. Biol. J. Linn. Soc. 78: 155-171.

1056 Machkewskyi V.K., Dmitrieva E.V., Gibson D.I., Al-Jufaili S. 2014. Lamellodiscus aff. euzeti 1057 Diamanka, Boudaya, Toguebaye \& Pariselle, 2011 (Monogenea: Diplectanidae) from the gills of 1058 Cheimerius nufar (Valenciennes) (Pisces: Sparidae) collected in the Arabian Sea, with comments 1059 on the distribution, specificity and historical biogeography of Lamellodiscus spp. Syst. Parasitol. 1060 89: 215-236.

1061 MacLeod N. 2013. Landmarks and semilandmarks: differences without meaning and meaning 1062 without differences. Available at: http://www.palass.org.

1063 Maddison W.P. 1997. Gene trees in species trees. Syst. Biol. 46: 523-536.

1064 Maechler M., Rousseeuw P., Struyf A., Hubert M., Hornik K. 2015. cluster: Cluster Analysis 1065 Basics and Extensions. R package version 2.0.2. Available at: https://cran.r1066 project.org/src/contrib/Archive/cluster/cluster_2.0.2.tar.gz.

1067 Marchiori N.C., Pariselle A., Pereira J., Agnese J.F., Durand J.D., Vanhove M.P. 2015. A

1068 comparative study of Ligophorus uruguayense and L. saladensis (Monogenea:

1069 Ancyrocephalidae) from Mugil liza (Teleostei: Mugilidae) in southern Brazil. Folia Parasitol. 62: $1070 \quad 024$.

1071 Marcus L.F. 1990. Traditional morphometrics. In: Rohlf F.J., Bookstein F.L., editors. Proceedings 1072 of the Michigan Morphometrics Workshop. Ann Arbor, Michigan: University of Michigan 1073 Museums. p.77-122. 
1074 Mariniello L., Ortis M., D’Amelio S., Petrarca V. 2004. Morphometric variability between and 1075 within species of Ligophorus Euzet \& Suriano, 1977 (Monogenea: Ancyrocephalidae) in the 1076 Mediterranean Sea. Syst. Parasitol. 57: 183-190.

1077 Mendlová M., Šimková A. 2014. Evolution of host specificity in monogeneans parasitizing 1078 African cichlid fish. Parasit. Vectors 7: 69.

1079 Messu Mandeng F.D., Bilong Bilong C.F., Pariselle A., Vanhove M., Bitja Nyom A.R., Agnèse J.1080 F. 2015. A phylogeny of Cichlidogyrus spp. (Monogenea, Dactylogyridea) clarifies a host-switch 1081 between fish families and reveals an adaptive component to attachment organ morphology of this 1082 parasite genus. Parasite. Vector. 8: 582.

1083 Minh B.Q., Nguyen M.A.T., von Haeseler A. 2013. Ultrafast approximation for phylogenetic 1084 bootstrap. Mol. Biol. Evol. 30: 1188-1195.

1085 Mollaret I., Jamieson B.G.M., Justine J.-L. 2000. Phylogeny of the Monopisthocotylea and 1086 Polyopisthocotylea (Platyhelminthes) inferred from 28S rDNA sequences. Int. J. Parasitol. 30: 1087 11-185.

1088 Morand S., Šimková A., Matejusová I., Plaisance L., Verneau O., Desdevises Y. 2002. 1089 Investigating patterns may reveal processes: evolutionary ecology of ectoparasitic monogeneans. 1090 Int. J. Parasitol. 32: 111-119. 
1091 Mouillot D., Šimková A., Morand S., Poulin R. 2005. Parasite species coexistence and limiting 1092 similarity: a multiscale look at phylogenetic, functional and reproductive distances. Oecologia 1093 146: 269-278.

1094 Mundry R. 2014. Statistical issues and assumptions of phylogenetic generalized least squares. In: 1095 Garamszegi L.Z., editor. Modern Phylogenetic Comparative Methods and Their Application in 1096 Evolutionary Biology. New York: Springer-Verlag. p. 131-153.

1097 Murray A.M. 2001. The fossil record and biogeography of the Cichlidae (Actinopterygii: 1098 Labroidei). Biol. J. Linn. Soc. 74: 517-532.

1099 Nieberding C.M., Olivieri I. 2007. Parasites: proxies for host genealogy and ecology? Trends 1100 Ecol. Evol. 22: 156-165.

1101 Nguyen L.-T., Schmidt H.A., von Haeseler A., Minh B.Q. 2015. IQ-TREE: A fast and effective 1102 stochastic algorithm for estimating maximum likelihood phylogenies. Mol. Biol. Evol. 32: 2681103274.

1104 Olson E.C., Miller E.L. 1958. Morphological Integration. Chicago: University of Chicago Press.

1105 Öztürk T., Özer A. 2014. Monogenean fish parasites, their host preferences and seasonal 1106 distributions in the Lower Kizilirmak Delta (Turkey). Turk. J. Fish. Aquat. Sc. 14: 367-378.

1107 Paradis E., Claude J., Strimmer K. 2004. APE: Analyses of Phylogenetics and Evolution in R 1108 language. Bioinformatics 20: 289-290.

1109 Pariselle A., Boeger W.A., Snoeks J., Bilong Bilong C.F., Morand S., Vanhove M.P.M. 2011. The 1110 monogenean parasite fauna of cichlids: a potential tool for host biogeography. Int. J. Evol. Biol. 1111 2011: 471480. 
1112 Perkins E.M., Donnellan S.C., Bertozzi T., Chisholm L.A., Whittington I.D. 2009. Looks can

1113 deceive: molecular phylogeny of a family of flatworm ectoparasites (Monogenea: Capsalidae)

1114 does not reflect current morphological classification. Mol. Phylogenet. Evol. 52: 705-714.

1115 Pepinelli M., Spironello M., Currie D.C. 2013. Geometric morphometrics as a tool for

1116 interpreting evolutionary transitions in the black fly wing (Diptera: Simuliidae). Zool. J. Linn.

1117 Soc. 169: 377-388.

1118 Pérez Ben C.M., Gómez R.O., Báez A.M. 2014. Intraspecific morphological variation and its

1119 implication in the taxonomic status of 'Bufo pisanoi', a Pliocene anuran from eastern Argentina.

1120 J. Vertebr. Paleontol. 34: 767-777.

1121 Pfennig D.W., Wund M.A., Snell-Rood E.C., Cruickshank T., Schlichting C.D., Moczek A.P. 1122 2010. Phenotypic plasticity's impacts on diversification and speciation. Trends Ecol. Evol. 25:

1123 459-467.

1124 Pizzo A., Zagaria D., Palestrini C. 2013. An unfinished speciation process revealed by geometric 1125 morphometrics, horn allometries and biomolecular analyses: The case of the fracticornis-similis1126 opacicollis species complex of the genus Onthophagus (Coleoptera: Scarabaeidae). Zool. Anz. 1127 252: 548-561.

1128 Poisot T., Desdevises Y. 2010. Putative speciation events in Lamellodiscus (Monogenea:

1129 Diplectanidae) assessed by a morphometric approach. Biol. J. Linn. Soc. 99: 559-569.

1130 Poisot T., Verneau O., Desdevises Y. 2011. Morphological and molecular evolution are not linked 1131 in Lamellodiscus (Plathyhelminthes, Monogenea). PLoS ONE 6: e26252.

1132 Poulin R. 2002. The evolution of monogenean diversity. Int. J. Parasitol. 32: 245-254. 
1133 R Core Team. 2015. R: A language and environment for statistical computing. R Foundation for 1134 Statistical Computing. Vienna, Austria. URL http://www/R-project.org/.

1135 Rae T.C. 1998. The logical basis for the use of continuous characters in phylogenetic systematics. 1136 Cladistics 14: 221-228.

1137 Raeymaekers J., Huyse T., Maelfait H., Hellemans B., Volckaert F. 2008. Community structure, 1138 population structure and topographical specialisation of Gyrodactylus (Monogenea) ectoparasites 1139 living on sympatric stickleback species. Folia Parasitol. 55: 187-196.

1140 Ramasamy P., Ramalingam K., Hanna R.E.B., Halton D.W. 1985. Microhabitat of gill parasites 1141 (Monogenea and Copepoda) of teleosts (Scomberoides spp.). Int. J. Parasitol. 15: 385-397.

1142 Reyment R.A., Blackith R.E., Campbell N.A. 1984. Multivariate Morphometrics. 2nd ed. 1143 London: Academic Press.

1144 Revell L.J. 2012. phytools: an R package for phylogenetic comparative biology (and other 1145 things). Methods Ecol. Evol. 3: 217-223.

1146 Revell L.J. 2014. Graphical methods for visualizing comparative data on phylogenies. In:

1147 Garamszegi L.Z., editor. Modern Phylogenetic Comparative Methods and Their Application in 1148 Evolutionary Biology. New York: Springer-Verlag. p. 77-104.

1149 Rodríguez-González A., Míguez-Lozano R., Llopis-Belenguer C., Balbuena J.A. $2015 a$.

1150 Phenotypic plasticity in haptoral structures of Ligophorus cephali (Monogenea: Dactylogyridae) 1151 on the flathead mullet (Mugil cephalus): a geometric morphometric approach. Int. J. Parasitol. 45: 1152 295-303. 
1153 Rodríguez-González A., Míguez-Lozano R., Llopis-Belenguer C., Balbuena J.A. 2015b. A new

1154 species of Ligophorus (Monogenea: Dactylogyridae) from the gills of the flathead mullet Mugil 1155 cephalus (Teleostei: Mugilidae) from Mexico. Acta Parasitol. 60: 767-776.

1156 Rohde K. 1977. A non-competitive mechanism responsible for restricting niches. Zool. Anz. 199:

$1157 \quad 164-172$.

1158 Rohde K. 1979. A critical evalution of intrinsic and extrinsic factors responsible for niche

1159 restriction in parasites. Am. Nat. 114: 648-671.

1160 Rohde K. 1994. Niche restriction in parasites - proximate and ultimate causes. Parasitology 109:

1161 S69-S84.

1162 Rohde K., Watson N. 1985. Morphology, microhabitats and geographical variation of Kuhnia

1163 spp. (Monogenea: Polyopisthocotylea). Int. J. Parasitol. 15: 569-586.

1164 Rohde K., Hobbs R.P. 1986. Species segregation: competition or reinforcement of reproductive

1165 barriers? In: Cremin M., Dobson C., Noorhouse E., editors. Parasites Lives: Papers on Parasites,

1166 Their Hosts and Their Associations to Honour JFA Sprent. St. Lucia: University of Queensland

1167 Press. p.189-199.

1168 Rohde K., Hayward C., Heap M., Gosper D. (1994). A tropical assemblage of ectoparasites - gill

1169 and head parasites of Lethrinus miniatus (Teleostei, Lethrinidae). Int. J. Parasitol. 24: 1031-1053.

1170 Rohlf F.J. 2013. tpsDig, digitize landmarks and outliers, version 2.17. Department of Ecology

1171 and Evolution, State University of New York at Stony Brook.

1172 Rohlf F.J. 2015. The tps series of software. Hystrix. 26, doi: 10.4404/hystrix-26.1-11264. 
1173 Rohlf F.J., Corti M. 2000. Use of two-block partial least-squares to study covariation in shape.

1174 Syst. Biol. 49: 740-753.

1175 Rohlf F.J., Marcus L.F. 1993. A revolution in morphometrics. Trends Ecol. Evol. 8: 129-132.

1176 Rohlf F.J., Slice D.E. 1990. Extensions of the Procrustes method for the optimal superimposition 1177 of landmarks. Syst. Zool. 39: 40-59.

1178 Roubal F.R. 1981. The taxonomy and site specificity of the metazoan ectoparasites on the black

1179 bream, Acanthopagrus australis (Günther), in northern New South Wales. Aust. J. Zool. 30: 11180100.

1181 Sanderson M.J., McMahon M.M., Steel M. 2010. Phylogenomics with incomplete taxon 1182 coverage: the limits to inference. BMC Evol. Biol. 10:155.

1183 Sarabeev V., Desdevises Y. 2014. Phylogeny of the Atlantic and Pacific species of Ligophorus 1184 (Monogenea: Dactylogyridae): morphology vs. molecules. Parasitol. Int. 63: 9-20.

1185 Shinn A.P., Gibson D.I., Sommerville C. 2001. Morphometric discrimination of Gyrodactylus 1186 salaris Malmberg (Monogenea) from species of Gyrodactylus parasitizing British salmonids 1187 using novel parameters. J. Fish Dis. 24: 83-97.

1188 Shinn A.P., Hansen H., Olstad K., Bachmann L., Bakke T.A. 2004. The use of morphometric 1189 characters to discriminate specimens of laboratory-reared and wild populations of Gyrodactylus 1190 salaris and G. thymali (Monogenea). Folia Parasitol. 51: 239:252.

1191 Sidlauskas B. 2008. Continuous and arrested morphological diversification in sister clades of 1192 characiform fishes: a phylomorphospace approach. Evolution 12: 3135-3156. 
1193 Sidlauskas B.L., Mol J.H., Vari R.P. 2011. Dealing with allometry in linear and geometric 1194 morphometrics: a taxonomic case study in the Leporinus cylindriformis group (Characiformes: 1195 Anostomidae) with description of a new species from Suriname. Zool. J. Linn. Soc. 162: 1031196130.

1197 Sievwright H., MacLeod N. 2012. Eigensurface analysis, ecology, and modelling of 1198 morphological adaptation in the falconiform humerus (Falconiformes: Aves). Zool. J. Linn. Soc. 1199 165: 390-419.

1200 Šimková A., Morand S. 2008. Co-evolutionary patterns in congeneric monogeneans: a review of 1201 Dactylogyrus species and their cyprinid hosts. J. Fish Biol. 73: 2210-2227.

1202 Šimková A., Rohde K. 2013. Community stability and instability in ectoparasites of marine and 1203 freshwater fish. In: Rohde K., editor. The Balance of Nature and Human Impact. Cambridge: 1204 Cambridge University Press. p. 75-88.

1205 Šimková A., Morand S. 2015. Parasite species coexistence and the evolution of the parasite niche. 1206 In: Morand S., Krasnov B.R., Littlewood D.T.J. editors. Parasite Diversity and Diversification. 1207 Cambridge: Cambridge University Press. p. 360-375.

1208 Šimková A., Ondračková M., Gelnar M., Morand S. 2002. Morphology and coexistence of 1209 congeneric ectoparasite species: reinforcement of reproductive isolation? Biol. J. Linn. Soc. 76: $1210125-135$.

1211 Šimková A., Verneau O., Gelnar M., Morand S. 2006. Specificity and specialization of 1212 congeneric monogeneans parazitizing cyprinid fish. Evolution 60: 1023-1037. 
1213 Šimková A., Serbielle C., Pariselle A., Vanhove M.P.M., Morand S. 2013. Speciation in 1214 Thaparocleidus (Monogenea: Dactylogyridae) parasitizing Asian pangasiid catfishes. BioMed 1215 Res. Int. Article ID 353956. doi:10.1155/2013/353956.

1216 Siquier G.F., Ostrowski de Núñez M. 2009. Ligophorus uruguayense sp. Nov. (Monogenea, 1217 Ancyrocephalidae), a gill parasite from Mugil platanus (Mugiliformes, Mugilidae) in Uruguay. 1218 Acta Parasitol. 54: 95-102.

1219 Smith U.E., Hendricks J.R. 2013. Geometric morphometric character suites as phylogenetic data: 1220 extracting phylogenetic signal from gastropod shells. Syst. Biol. 62: 366-385.

1221 Soo O.Y.M., Lim L.H.S. 2012. Eight new species of Ligophorus Euzet \& Suriano, 1977

1222 (Monogenea: Ancyrocephalidae) from mugilids off Peninsular Malaysia. Raffles Bull. Zool. 60: 1223 241-264.

1224 Soo O.Y.M., Lim L.H.S. 2015. A description of two new species of Ligophorus Euzet \& Suriano, 12251977 (Monogenea: Ancyrocephalidae) from Malaysian mugilid fish using principal component 1226 analysis and numerical taxonomy. J. Helminthol. 89: 131-149.

1227 Soo O.Y.M., Tan W.B., Lim L.H.S. 2015. Three new species of Ligophorus Euzet \& Suriano, 1228 1977 (Monogenea: Ancyrocephalidae) from Moolgarda buchanani (Bleeker) off Johor, Malaysia 1229 based on morphological, morphometric and molecular data. Raffles Bull. Zool. 63: 49-65.

1230 Sparks J.S., Smith W.L. 2005. Freshwater fishes, dispersal ability, and non-evidence: “Gondwana 1231 Life Rafts" to the rescue. Syst. Biol. 53: 11-19.

1232 Swofford D.L. 2002. PAUP: phylogenetic analysis using parsimony, Ver. 4.0.b10. Sunderland, 1233 Massachusetts: Sinaeur Associates. 
1234 Tamura K., Stecher G., Pedersen D., Filipski A., Kumar S. 2013. MEGA 6: Molecular

1235 Evolutionary Genetics Analysis Version 6.0. Mol. Biol. Evol. 30: 2725-2729.

1236 Tan W.B. 2013. Morphological and molecular characterisation of monogeneans. Ph.D thesis, 1237 University of Malaya.

1238 Tan W.B., Khang T.F., Lim L.H.S. 2010. Morphometric analysis of Trianchoratus Price \& Berry, 12391966 (Monogenea: Heteronchocleidinae) from Channa spp. (Osteichthyes: Channidae) of 1240 Peninsular Malaysia. Raffles Bull. Zool. 58: 165-172.

1241 Teo B.G., Sarinder K.K.S., Lim L.H.S. 2010. A novel alternative method for 3D visualisation in 1242 parasitology: the construction of a 3D model of a parasite from 2D illustrations. Trop. Biomed. $1243 \quad 27: 254-264$.

1244 Teo B.G., Sarinder K.K.S., Lim L.H.S. 2013. A deformable generic 3D model of haptoral anchor 1245 of monogenean. PLoS ONE 8(10): e77650.

1246 Thiele K. 1993. The holy grail of the perfect character: the cladistic treatment of morphometric 1247 data. Cladistics 9: 275-304.

1248 van Valen L. 1965. Morphological variation and width of ecological niche. Am. Nat. 99: 377-390.

1249 Vanhove M.P.M., Huyse T. 2015. Host specificity and species jumps in fish-parasite systems. In:

1250 Morand S., Krasnov B.R., Littlewood D.T.J. editors. Parasite Diversity and Diversification.

1251 Cambridge: Cambridge University Press. p. 401-419.

1252 Vanhove M.P.M., Tessens B., Schoelinck C., Jondelius U., Littlewood D.T.J., Artois T., Huyse T.

1253 2013. Problematic barcoding in flatworms: A case-study on monogeneans and rhabdocoels

1254 (Platyhelminthes). ZooKeys 365: 355-379. 
1255 Vanhove M.P.M., Pariselle A., Van Steenberge V., Raeymaekers J.A.M., Hablützel P.I., Gillardin

1256 C., Hellemans B., Breman F.C., Koblmüller S., Sturmbauer C., Snoeks J., Volckaert F.A.M., 1257 Huyse T. 2015. Hidden biodiversity in an ancient lake: phylogenetic congruence between Lake

1258 Tanganyika tropheine cichlids and their monogenean flatworm parasites. Sci. Rep. 5: 13669.

1259 Vignon M. 2011. Putting in shape - towards a unified approach for the taxonomic description of 1260 monogenean haptoral hard parts. Syst. Parasitol. 79: 161-174.

1261 Vignon M., Sasal P. 2010. The use of geometric morphometrics in understanding shape

1262 variability of sclerotized haptoral structures of monogeneans (Platyhelminthes) with insights into 1263 biogeographic variability. Parasitol. Int. 59: 183-191.

1264 Vignon M., Pariselle A., Vanhove M.P.M. 2011. Modularity in attachment organs of African 1265 Cichlidogyrus (Platyhelminthes: Monogenea: Ancyrocephalidae) reflects phylogeny rather than 1266 host specificity or geographic distribution. Biol. J. Linn. Soc. 102: 694-706.

1267 Waeschenbach A., Webster B.L., Bray R.A., Littlewood D.T.J. 2007. Added resolution among 1268 ordinal level relationships of tapeworms (Platyhelminthes: Cestoda) with complete small and 1269 large subunit nuclear ribosomal RNA genes. Mol. Phylogenet. Evol. 45: 311-325.

1270 Warnes G.R., Bolker B., Bonebakker L., Gentleman R., Huber W., Liaw A., Lumley T., Maechler 1271 M., Magnusson A., Moeller S., Scharwtz M., Venables B. 2014. gplots: various R programming 1272 tools for plotting data. R package version 2.13.0. Available at: http://CRAN.R1273 project.org/pacakge $=$ gplots.

1274 Werneburg I., Wilson L.A.B., Parr W.C.H., Joyce W.G. 2015. Evolution of neck vertebral shape 1275 and neck retraction at the transition to modern turtles: an integrated geometric morphometric 1276 approach. Syst. Biol. 64: 187-204. 
1277 Wheeler Q.D. 2007. Digital innovation and taxonomy’s finest hour. In: MacLeod N., editor.

1278 Automated Taxon Identification in Systematics: Theory, Approaches and Applications. Boca 1279 Raton: CRC Press. p. 9-23.

1280 Whittington I.D. 2005. Monogenea Monopisthocotylea (ectoparasitic flukes). In: Rohde K., 1281 editor. Marine Parasitology. Australia: CSIRO Publishing. p. 63-72.

1282 Whittington I.D., Cribb B.W., Hamwood T.E., Halliday J.A. 2000. Host-specificity of 1283 monogenean (platyhelminth) parasites: a role for anterior adhesive areas? Int. J. Parasitol. 30: $1284 \quad 305-320$.

1285 Whittington I.D., Kearn G.C. 2011. Hatching strategies in monogenean (platyhelminth) parasites 1286 that facilitate host infection. Integr. Comp. Biol. 51: 91-99.

1287 Wiens J.J. 2001. Character analysis in morphological phylogenetics: problems and solutions. 1288 Syst. Biol. 50: 689-699.

1289 Wilkinson L., Friendly M. 2008. The history of the cluster heat map. Am. Stat. 63: 179-184.

1290 Wong W.L., Gorb S.N. 2013. Attachment ability of a clamp-bearing fish parasite, Diplozoon 1291 paradoxum (Monogenea) on gills of the common bream Abramis brama. J. Exp. Biol. 216: 300812923014.

1293 Yang Z. 2006. Computational Molecular Evolution. Oxford, UK: Oxford University Press.

1294 Zietara M.S., Lumme J. 2002. Speciation by host switch and adaptive radiation in a fish parasite 1295 genus Gyrodactylus (Monogenea, Gyrodactylidae). Evolution 56: 2445-2458.

1296 FIGURE CAPTIONS 
1297 FIGURE 1. a) Landmarks of the i) ventral and ii) dorsal anchors of a L. navjotsodhii sample, 1298 digitized using TPSDIG2 (Version 2.17). The iii) ventral and iv) dorsal bars can also be seen in 1299 the image. b) Landmark positions on an anchor. Type I landmarks 1300 (LM1,LM2,LM3,LM5,LM7,LM8) are indicated by stars, while Type III landmarks 1301 (LM4,LM6,LM9,LM10,LM11) are indicated by solid circles. Abbreviations: ORP - outer root 1302 point; GP - groove point; IRP - inner root point; DP - dent point; CP - curve point; TP - tip point. 1303 FIGURE 2. Wireframe plots of anchors of L. navjotsodhii lying in their natural positions in the 1304 mounted slide. a) Example of a poor quality specimen $(\mathrm{Q}=5)$; note the larger than expected 1305 variation between shape of (larger) left and right forms of the dorsal anchors, which shows up in 1306 a Tukey Mean Difference (TMD) plot that has relatively wide width of $95 \%$ limits of agreement 1307 (upper and lower dashed lines) as well as a fanning pattern as $A$ becomes larger. b) Example of a 1308 good quality specimen $(\mathrm{Q}=30)$; note the lack of shape variation between left and right forms of 1309 ventral and dorsal anchors, which shows up in a TMD plot with much narrower width of 95\% 1310 limits of agreement as well as a more or less random deviation of $M$ about 0 independent of $A$.

1311 FIGURE 3. Molecular phylogeny of the 13 Ligophorus species inferred using the maximum 1312 likelihood method (10000 bootstrap replicates) with annotations from three anatomical structures: 1313 anchors, bars and male copulatory organ (30\% of original scale). Species in Clade I are found in 1314 Moolgarda buchanani, and species in Clade II are found in Liza subviridis. The ventral and 1315 dorsal forms of the anchors are arranged from left to right, those of the bars from top to bottom.

1316 FIGURE 4. Scatter plots of Generalized Procrustes Analysis coordinates of all specimens for the 1317 a) ventral anchors and b) dorsal anchors. The species centroids of each landmark are indicated by 1318 solid, colored circles. 
1319 FIGURE 5. PCA plots of PC1 against PC2 and PC3 for a) ventral and b) dorsal anchors, with 1320 superimposed phylogeny of the 13 Ligophorus species. The centroids of the species are indicated 1321 in solid colors, while individual samples are plotted in high transparency colors. The estimated 1322 principal component coordinates of the ancestral nodes are represented by small open circles.

1323 FIGURE 6. Cluster heat map of specimens (column) using shape variable data (row). The name 1324 of a shape variables consists of three parts: a prefix indicating ventral (V) or dorsal (D) anchors, a 1325 number indicating landmark index, and a suffix indicating x or y coordinate value. a) Cluster heat 1326 map using filtered specimens with quality score of 10 or more $(n=437)$; b) Cluster heat map using 1327 all specimens $(\mathrm{n}=530)$.

FIGURE 7. Wireframe-lollipop plots of mean shape change relative to estimated ancestral mean shape in a) ventral anchors of Clade I (purple); b) dorsal anchors of Clade I; c) ventral anchors of Clade II (blue); d) dorsal anchors of Clade II. The p-value of Rayleigh test for uniform direction change at each landmark is indicated as a colored solid circle. The color bar maps color tones to their corresponding p-values.

FIGURE 8. Scatter plots LM1-LM5 distance against LM1-LM3 distance for a) ventral and b) dorsal anchors. The dashed lines are cut-offs on the $\mathrm{x}$ and $\mathrm{y}$ axes that allow complete discrimination of Clade I from Clade II. Scatter plots of outer length (OL) against inner length (IL) for c) ventral and d) dorsal anchors. No cut-offs on the $\mathrm{x}$ and $\mathrm{y}$ axes permit complete discrimination of Clade I from Clade II.

FIGURE 9. Continuous character mapping of anchor size (in $\mu \mathrm{m}$ ) of ventral (left) and dorsal 1339 (right) anchors onto the maximum likelihood phylogeny of the 13 Ligophorus species. 
1342 similar, species that have similar size for the male copulatory organ show variation in the latter's 1343 morphology (I and II), whereas those with similar morphology of male copulatory organ show 1344 variation in the latter's size (III).

1345 FIGURE 11. Color-coded morphological character state data for the 13 Ligophorus species and 1346 their estimated maximum parsimony phylogeny (1000 bootstrap replicates). a) Result using the 1347 set of morphological characters (7 to 12) that contain discretized LM1-LM3 and LM1-LM5 1348 distances and anchor shaft shape. b) Result using anchor morphological characters derived from 1349 traditional morphometrics in Sarabeev and Desdevises (2014).

1350 TABLE CAPTIONS

1351 TABLE 1. GenBank accession numbers of 28S rRNA, 18S rRNA and ITS1 sequences of the 13 1352 Ligophorus species, with information about the latters' host species and collection location.

1353 Sequences obtained in present study are marked with an asterisk.

1354 TABLE 2. List of morphological characters used to construct maximum parsimony trees. All 1355 characters in Set B were taken from Sarabeev and Desdevises (2014). Characters 1-6 of Set B are 1356 the same as Set A's; Characters 7-12 of Set A were constructed in the present study based on 1357 results of geometric morphometric analysis. 